\title{
LA-UR $94-1544$
}

TITLE: RCRA METALS ANALYSIS BY LASER-INDUCED BREAKDOWN SPECTROSCOPY: DETECTION LIMITS IN SOILS

AUTHOR(S): Aaron Koskelo, CST-4

David A. Cremers, CST-2

\author{
SUBMITTEO TO: Caroline Purdy, DOE, EM-541 \\ Characterization, Monitoring and Sensor Technology - \\ Integrated Program
}

\section{DISCLAIMER}

\begin{abstract}
This report was prepared as an account of work sponsored by an agency of the United States Government. Neither the United States Government nor any agency thereof, nor any of their employees, makes any warranty, express or implied, or assumes any legal liability or responsibility for the accuracy, completeness, or usefulness of any information, apparatus, product, or process disclosed, or represents that its use would not infringe privately owned rights. Reference herein to any specific commercial product, process, or service by trade name, trademark, manufacturer, or otherwise does not necessarily constitute or imply its endorsement, recommendation, or favoring by the United States Government or any agency thereof. The views and opinions of authors expressed herein do not necessarily state or reflect those of the United States Government or any agency thereof.
\end{abstract}

By acceptance of this article. the publisher recognizes that the U.S Government retains a nonexclusive. rayalty-free license to publish or reproduce the published form of this contribution or 10 altow others 1000 so. for U.S. Government purposes. 


\section{DISCLAIMER}

Portions of this document may be illegible in electronic image products. Images are produced from the best available original document. 


\section{RCRA Metals Analysis by Laser-Induced Breakdown Spectroscopy \\ Detection Limits in Soils}

\section{Aaron Koskelo and David A. Cremers \\ Chemical Sciences and Technology Division Los Alamos National Laboratory}

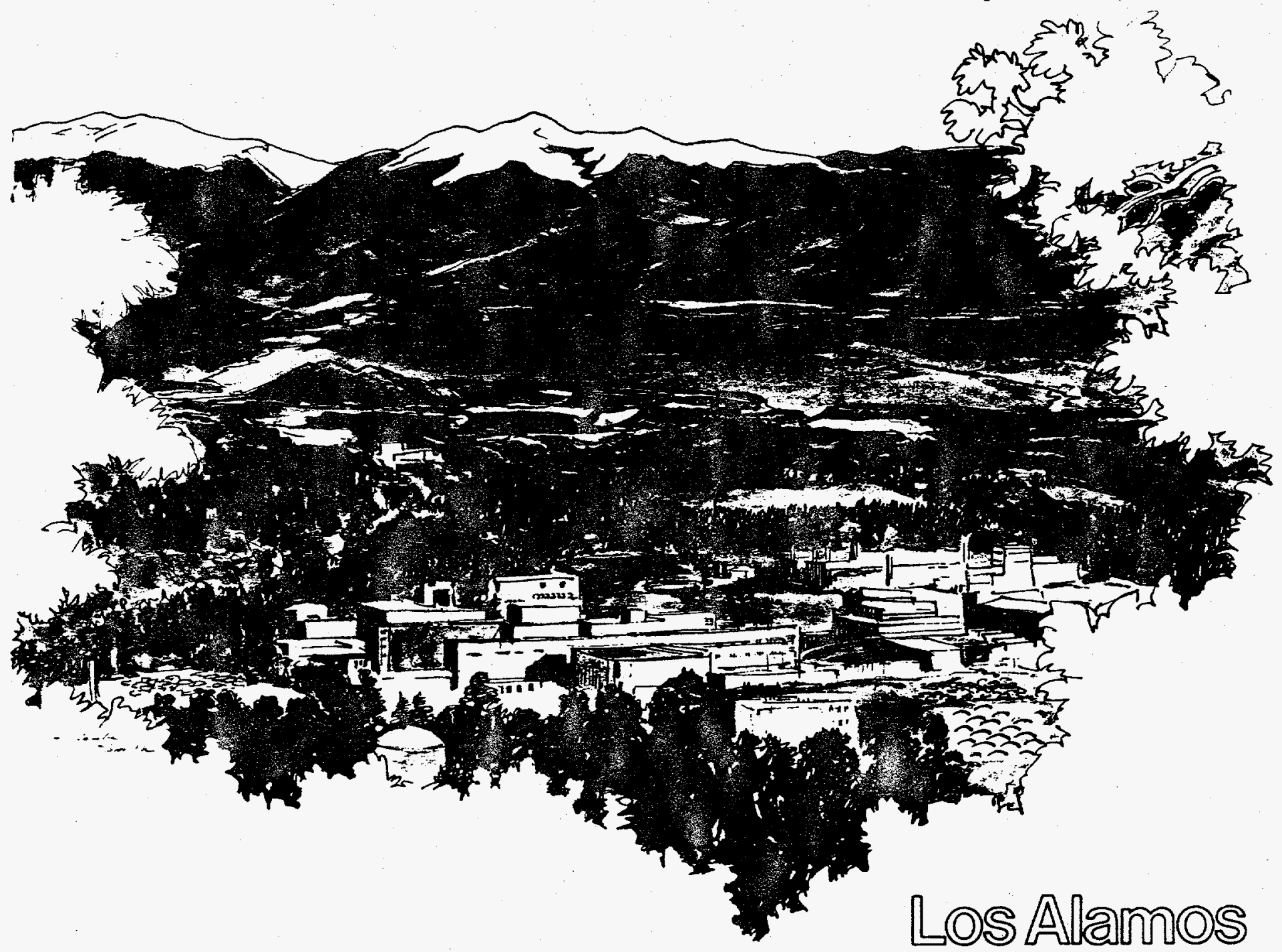

Los Alamos National Laboratory Los Alamos, New Mexico 87545 


\section{RCRA METALS ANALYSIS BY LASER-INDUCED BREAKDOWN SPECTROSCOPY}

\section{Detection Limits in Soils}

Aaron Koskelo and David A. Cremers

Chemical Sciences and Technology Division

Los Alamos National Laboratory 


\section{Acknowledgments}

The authors wish to cite the considerable contributions of the research team assembled for this work at LANL. Much of the data in this report were acquired by Thomas Gamble. Aaron Eppler contributed the work on barium and assisted with the scanning electron microscopy. Leeann Foster contributed the data for beryllium. Thomas Kos made most of the spectral assignments.

This work is funded through the Characterization, Monitoring and Sensor Technology Integrated Program, DOE EM-541. 


\section{Introduction}

The goal of the Technical Task Plan (TTP) that this report supports is research, development, testing and evaluation of a portable analyzer for RCRA and other metals. The instrumentation to be built will be used for field-screening of soils. Data quality is expected to be suitable for this purpose. The data presented in this report were acquired to demonstrate the detection limits for laser-induced breakdown spectroscopy (LIBS) of soils using instrument parameters suitable for fieldable instrumentation. The data are not expected to be the best achievable with the high pulse energies available in laboratory lasers.

The instrumentation will be based on the method of laser-induced breakdown spectroscopy [1]. In the LIBS method, sample atomization and excitation is provided directly by a laser spark. Time consuming and hazardous sample preparation is eliminated. The spark is produced by focusing pulses of laser light on the material to be analyzed to generate a small microplasma. Because plasma temperatures initially. exceed $10,000 \mathrm{~K}$, the material in the plasma is efficiently vaporized, reduced to its elemental constituents, and the resulting atoms electronically excited.

Advantages of LIBS for field-based analyses of soils are:

1) Materials can be analyzed directly in the field. Little or no sample preparation is required. Adulteration of the sample by improper handling is minimized or eliminated entirely.

2) Rapid analysis. A complete spectrum can be obtained in a single laser shot. The technique is as rapid as the repetition rate of the laser and detection apparatus allow.

3) Simplicity. Skilled operators are not required.

4) Portability. Lasers with the necessary pulse energy for LIBS that weigh less than two pounds are now available. Compact detection systems are also available. One complete apparatus developed in our laboratory, including power supplies, detection apparatus and a portable computer is the size of a small suitcase. The active sampling unit, which includes the laser head and optics is handheld.

5) Simultaneous multi-element analysis. All elements in the microplasma are electronically excited and can be detected simultaneously, in principle.

6) Spatial discrimination. The analytical region for LIBS from a single laser pulse is the size of the induced spark. In practice, with lasers of convenient size, this region can be as small as $.01 \mathrm{~mm}^{3}$ or as large as $0.5 \mathrm{~mm}^{3}$. Larger regions are analyzed by rastering of the laser beam.

7) Remote detection. The spark is produced by focused optical radiation. Thus, only optical access to the sample is required. In work to be done for this TTP, the utility of delivery of the laser light using a fiber optic cable will be investigated. In other work we are conducting, we believe LIBS spectra can be obtained at distances as great as 80 feet from the material to be analyzed.

8) Sensitivity. Detection limits should be suitable for field-screening data for many elements of interest.

This report presents our work to date on the detection limits for several elements in soils using LIBS. The elements targeted in the Technical Task Plan are antimony, arsenic, beryllium, cadmium, chromium, lead, selenium and zirconium. Data for these elements are presented in this report. We also have included other data of interest to potential customers for the portable LIBS apparatus. These data are for barium, mercury, cesium and strontium. Data for uranium and thorium will be acquired during the tasks geared toward mixed waste characterization.

Table 1 summarizes our results. 
Table 1. Summary of $3 \mathrm{~s}$ detection limits for selected elements in soils using laserinduced breakdown spectroscopy. Instrument parameters are as noted. Concentrations using X-ray fluorescence spectroscopy were obtained by Reference 5 . 


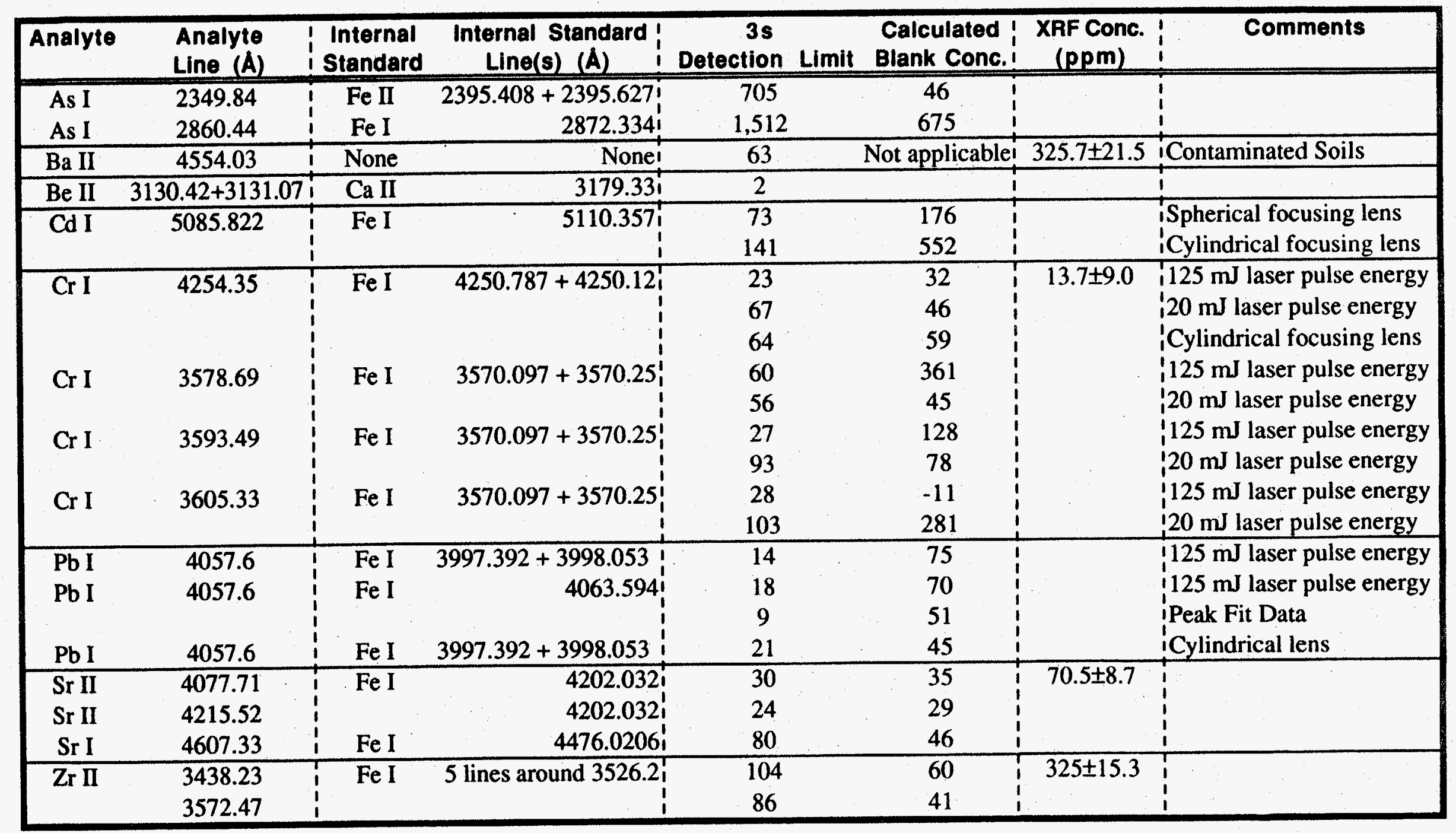




\section{Experiment}

A schematic of the LIBS apparatus is shown in Figure 1. The laser used was a Quanta Ray DCR-11. This laser has donut-mode beam profile in the far-field. The energy of the laser pulses was kept at $125 \mathrm{~mJ}$ for all data except where noted. A $100 \mathrm{~mm}$ focal length spherical lens was used to focus the laser beam on the surface of the soil sample pellets for most of this work. For some experiments, the spherical lens was replaced with a $100 \mathrm{~mm}$ focal length cylindrical lens to create a long spark. In both cases, a motor-driven translation stage moved the pellet under the laser beam to present a fresh surface on every laser shot. Figure 2 is a picture obtained with a $100 \mathrm{x}$ microscope showing the track left by the laser. Under the conditions used it is clear that the large underlying crystalline structure of the soil largely remains intact. The laserinduced plasma is created from the material that resides on the surface of the soil particles.

Samples were prepared as follows. Local soil was gathered by removing the top two inches of the soil surface with a shovel, including the vegetative layer.

Approximately 1 liter was collected. This soil was passed through a series of sieves. The fraction passing through a number 25 sieve, (nominal aperture size is $710 \mu \mathrm{m}$ ), was retained for the spiked samples.

Spiked samples were prepared by the following procedure. All glassware was cleaned using the standard EPA cleaning procedure except Type II water was used for rinsing instead of tap water. Aliquots of atomic absorption standards were diluted with acid to $10 \mathrm{ml}$ in a volumetric flask. Acid solutions used for dilution were prepared to match the matrix of the undiluted standard. These acids were either $0.3 \mathrm{M} \mathrm{HCl}$ or $0.3 \mathrm{M}$ $\mathrm{HNO}_{3} .10 \mathrm{~g}$ of sieved soil was transferred to a beaker ( 20 or $50 \mathrm{ml}$ total volume). The diluted aliquot was added to the beaker and stirred to completely wet the soil. The volumetric flask was then refilled with $10 \mathrm{ml}$ of the appropriate acid and this rinse was added to the beaker. Most samples were then completely dried in a $40^{\circ} \mathrm{C}$ drying oven. The drying period was 48 hours. The $\mathrm{Sb}, \mathrm{Hg}$ and As samples were air dried under ambient conditions in a fume hood. Drying of these three classes of samples took 4 days.

After drying, each $10 \mathrm{~g}$ sample was ground by hand with a porcelain mortar and pestle. $4 \mathrm{~g}$ of the sample was pressed into a pellet using 20 tons of pressure applied for 10 minutes. No binder was used. The unused $6 \mathrm{~g}$ of soil was stored in a screw cap polyethylene container for archival purposes.

A survey was conducted using a Los Alamos soil sample, NIST soil samples (NIST Standard Reference Materials 2709, 2710 and 2711) and a sample containing $3.57 \%$ antimony and $0.66 \%$ arsenic (Canadian Certified Reference Materials Project, Antimony Ore CD-1),

Reference lines were chosen from those iron lines which clearly have no spectral interference and appear in the same spectrum as the analyte. Primary interfering elements are titanium and chromium. Since many of the transitions involved in the analyte lines have unknown upper energy levels, no attempt was made to match the excited state energy levels of the iron and that of the analyte. Small improvements in precision might be obtained by a careful choice of the reference line.

Spectra were calibrated in wavelength by acquiring LIBS spectra of iron (Bureau of Analyzed Samples, Ltd., Spectroscopic Standard SS50B). In some cases, metallic lead, copper and aluminum were also used to calibrate wavelengths. Two different gratings were used in the 1 meter spectrograph depending on the wavelength. A 3600 $1 / \mathrm{mm}$ grating was used for some of the data and gave wavelength accuracies of $\leq 0.15 \AA$. With a $12001 / \mathrm{mm}$ grating accuracy of the wavelength calibration was found to be $\leq 0.4 \AA$. Iron lines were identified in the soil spectra by comparison with the LIBS spectrum of pure iron.

Calibration curves were prepared by using the net integrals. LIBS spectra of samples ranging from 0 to $1000 \mathrm{ppm}$ were used. In the case of arsenic, the range ran to 
$10,000 \mathrm{ppm}$. Detection limits were calculated from the slope of the calibration curve and using the standard deviation of the blank. The blank was prepared in the same manner as the spiked soil samples.

As a general rule, the precision of the measurements stays within a narrow range as a function of concentration for samples above the detection limit. For concentrations decreasing below the calculated detection limit, the precision also decreases. This indicates that inhomogeneously distributed material is the primary source of noise at low sample concentrations. This is not surprising given the small sample volume of the laser spark. Standard laboratory methods of analysis eliminate this by homogenizing the sample taken from a relatively large volume. The homogenization effects a mechanical signal averaging. Since LIBS measures the distribution of analyte on a microscopic scale, the averaging must be done computationally. Figure 3 illustrates the distribution of trace analytes in NIST Reference Soil 2710 observed by scanning electron microscopy. The white areas are deposits of various lead, barium and zinc compounds. A survey of other areas with the scanning electron microscope showed that the trace elements reside in discrete, concentrated sites as in the figure. 
Figure 1. Schematic of the laboratory LIBS apparatus. The sample is mounted on a motorized translation stage and translated as the laser fires. 


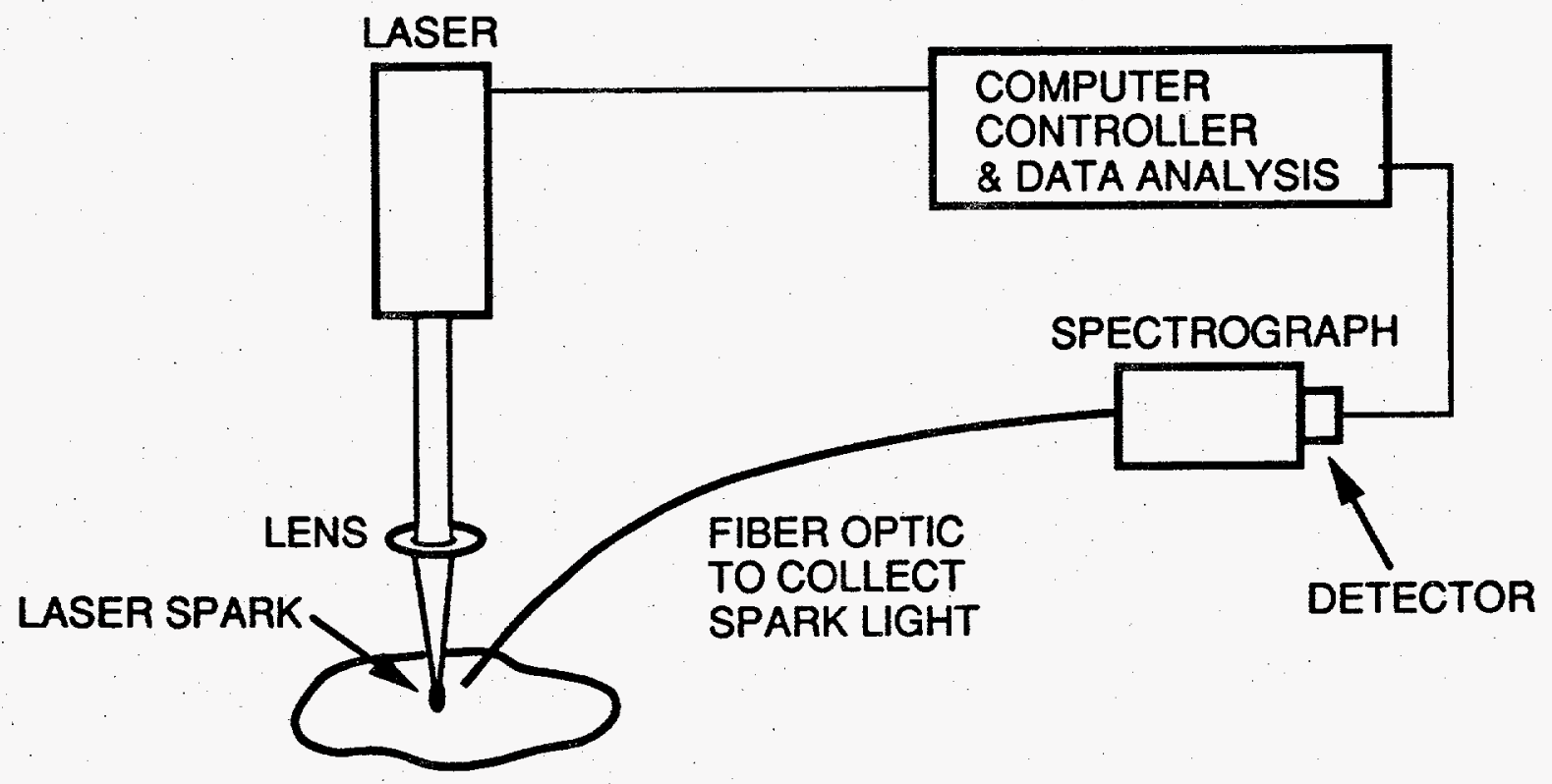

Figure 1. 
Figure 2. A microscopic view of the track left by the laser spark in soil. The smallest division of the superimposed scale is .002". 


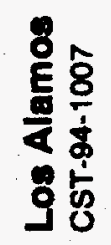

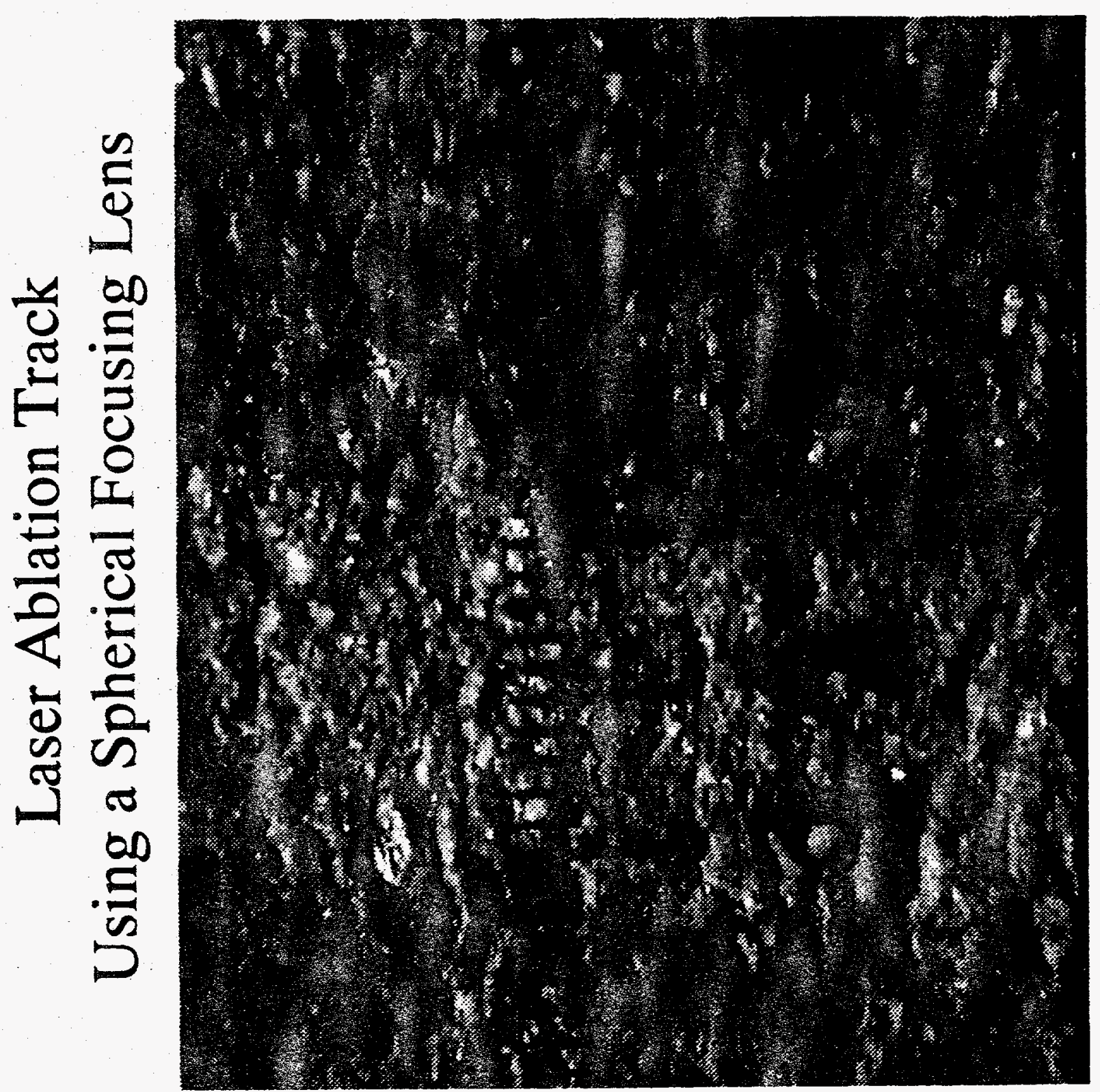


Figure 3. Scanning electron micrograph of a thin section prepared from NIST Standard Reference Material 2710. The large triangular piece at the lower right is feldspar. Most of the material that appears in white contains lead phosphate. Some arsenic and zinc also are present. The substrate for the piece towards the upper left, which has heavy surface contamination, appears to be quartz. 


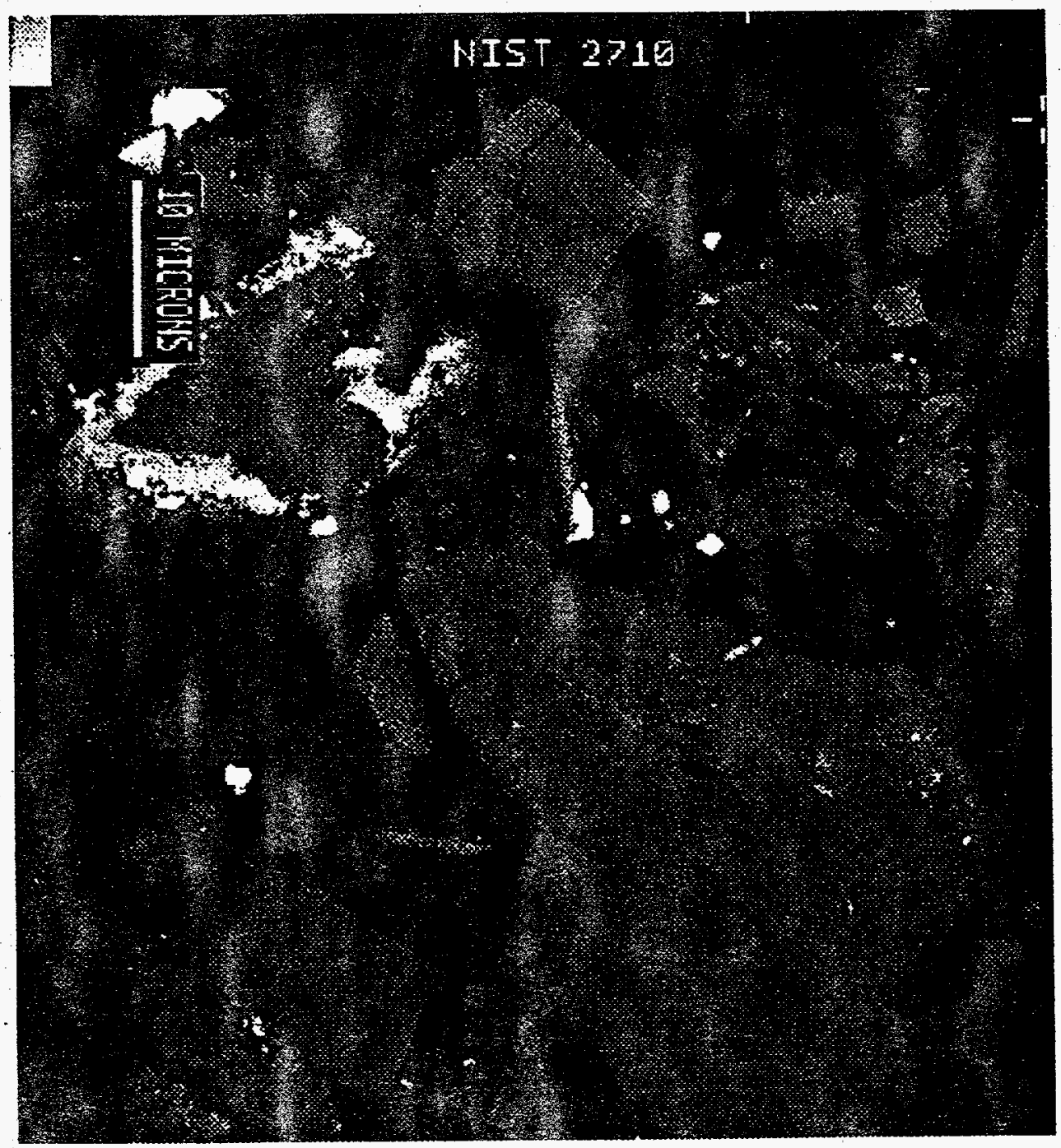




\section{ARSENIC}

The most intense lines for arsenic listed in the NIST data tables are ionic lines [2]. We have not observed these lines in our survey scans. This is due to either spectral interference, low line sensitivity, or both, depending on the line. Also, because of the low sensitivity of the instrumentation used below about $2300 \AA$, the atomic lines commonly used in ICP $(1890.4,1937.6,1937.0,1972.0$ and $2288.1 \AA)$ could not be observed [3]. In addition, the $2288.1 \AA$ As I line is unsuitable for use in the presence of high cadmium conce atrations. In anticipation of the necessity of observing arsenic in the presence of other RCRA elements, the $2288.1 \AA$ line was not chosen for further investigation.

We have observed several atomic lines. Observed were: $2288.12 \AA, 2349.84 \AA$, $2780.22 \AA$ and $2860.44 \AA$. In addition, we observed As II at $2830.359 \AA, 2831.164 \AA$ and $2884.406 \AA$. The first two lines of these were too weak to be useful; the $2884.406 \AA$ line has a strong nearby interference from Fe II $2883.702 \AA$.

With these considerations, two As I lines were used to prepare calibration curves. These were the $2349.84 \AA$ and $2860.44 \AA$ lines.

Figure 4 shows a LIBS spectrum obtained from Los Alamos Soil spiked with arsenic to give $999 \mathrm{ppm}$ (+ blank concentration). Figure 5 shows the spectral region of the Fe II lines used for the internal reference. The peak labeled Fe II $2395.627 \AA$ may also have intensity from Fe II $2395.408 \AA$.

Figure 6 is a calibration curve for the As I $2349.84 \AA$ line using the Fe II lines as a reference. The calibration curve is linear except for some possible curvature at $10,000 \mathrm{ppm}$. Using the slope of a linear fit to the points, the calculated $3 \mathrm{~s}$ detection limit is $705 \mathrm{ppm}$.

The relative standard deviations of the points between $1000 \mathrm{ppm}$ and $10,000 \mathrm{ppm}$ range between $10 \%$ to $21 \%$. The relative standard deviation for the $300 \mathrm{ppm}$ sample, (which has a concentration below the detection limit), is much greater. Detector noise is 0.084 counts absolute using the same region of interest as the arsenic line. The noise on the signal for the $300 \mathrm{ppm}$ sample is 1.74 counts absolute; for the blank, 1.88 counts. The primary source of noise is not the detector. Rather it comes from fluctuations in the plasma emission.

The samples were made by spiking the soil with aliquots of known amounts of analyte. The method of standard additions can be used to calculate the amount of arsenic in the blank that is present in the same matrix as the added analyte. Doing so, we obtain a value of $47 \mathrm{ppm}$ As in the Los Alamos soil samples. This is above the general range of concentrations found in the soils in this area of 1.2 to $11 \mathrm{ppm}$, as determined by laboratory X-ray fluorescence spectroscopy [4].

A calibration curve for As I $2860.44 \AA$ using Fe I 2872.334 $\AA$ as an internal reference resulted in a detection limit of $1500 \mathrm{ppm}$. 
Figure 4. LIBS spectrum of soil near As I 2349.84 $\AA$. The upper trace was obtained from a sample spiked with arsenic. The net concentration of the spiked soil is $999 \mathrm{ppm}$ plus background. The lower trace is the spectrum obtained from a soil blank. 


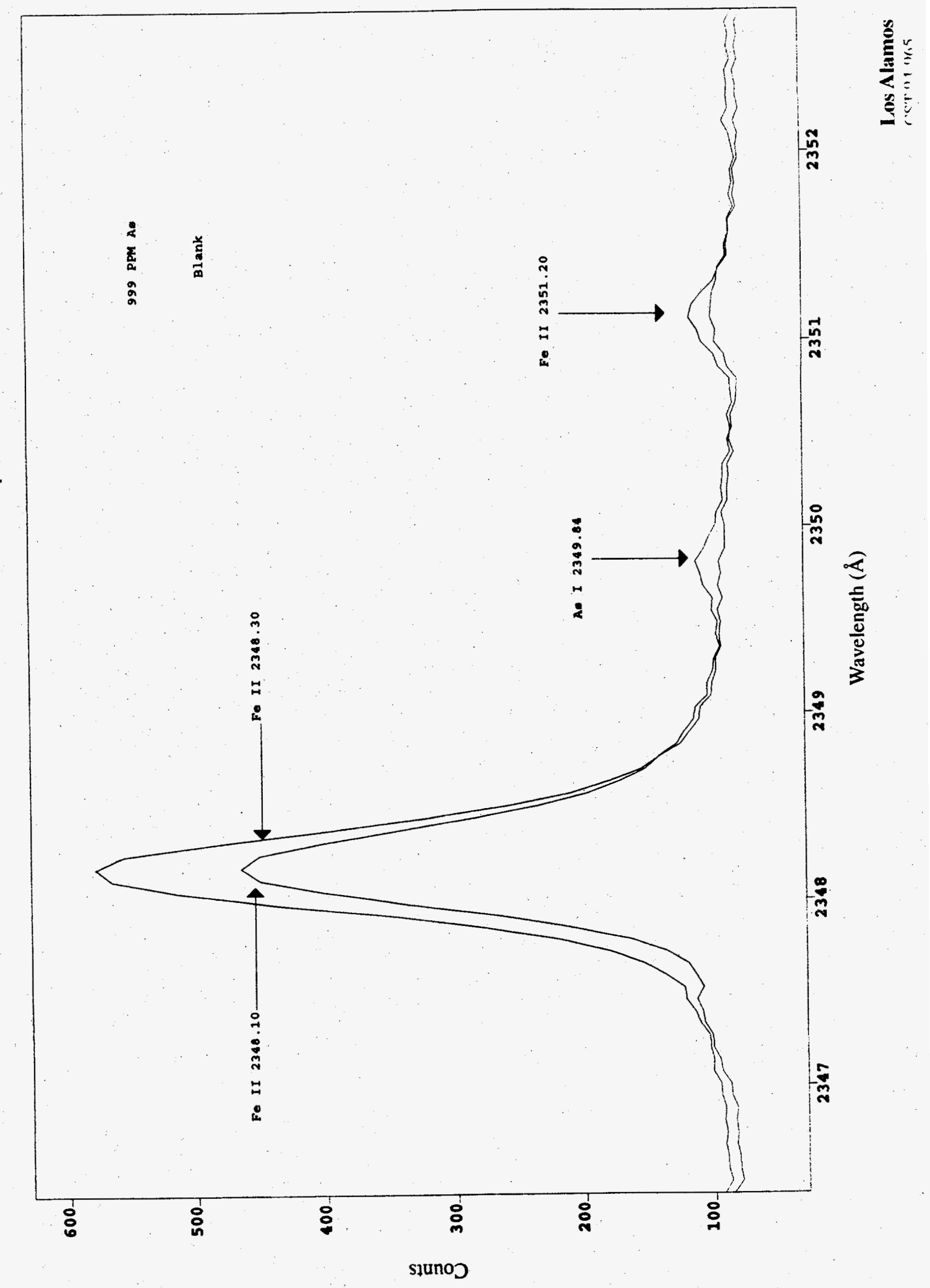


Figure 5. LIBS spectrum of soil near Fe II $2395.627 \AA$. This spectrum is an expansion of the same spectrum used for figure 4 . The upper trace is the spectrum obtained from the soil blank. The lower trace was obtained from the sample spiked with arsenic. The iron "line" is actually an unresolved pair of lines at $2395.408 \AA$ and $2395.627 \AA$. 


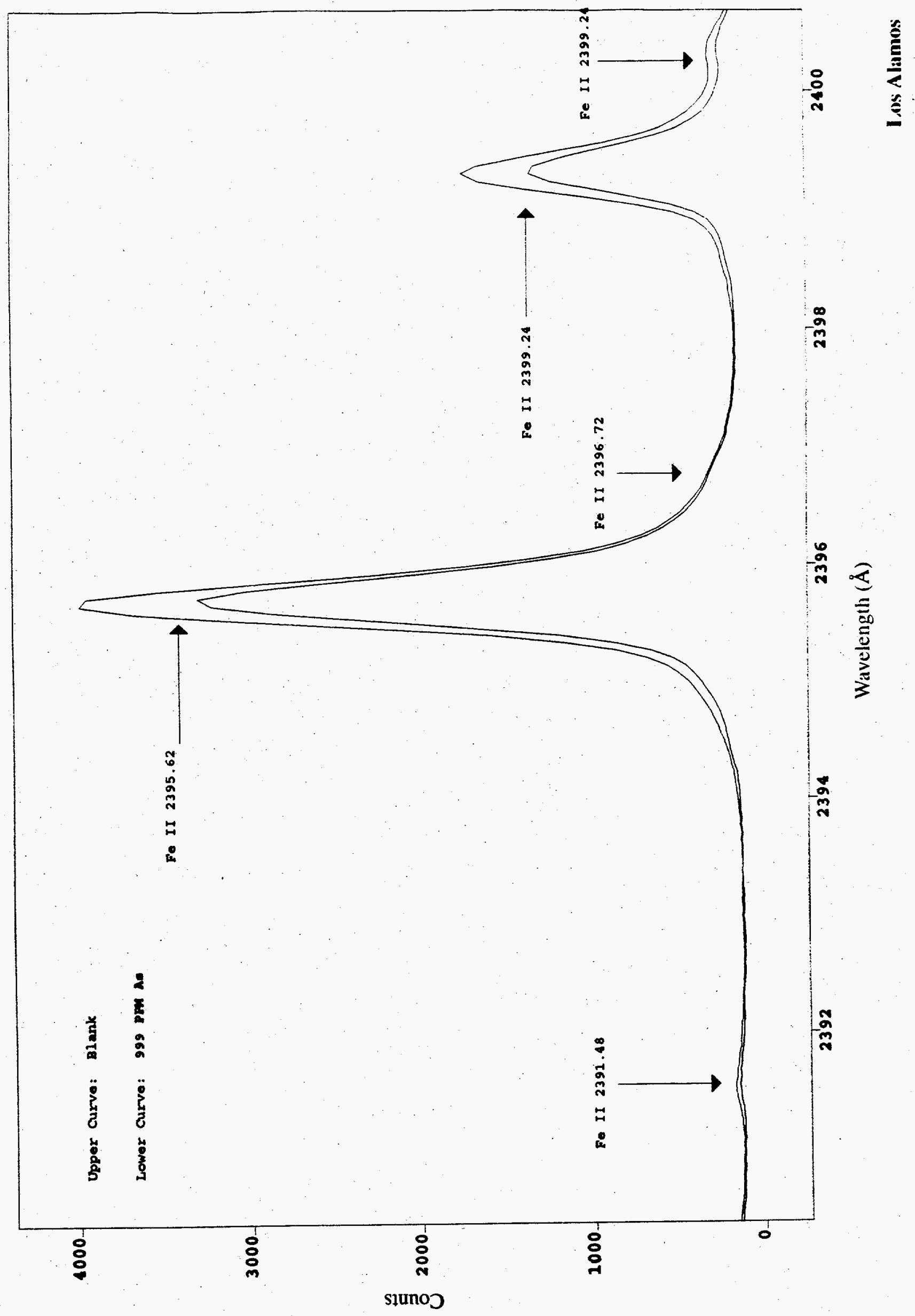


Figure 6. Calibration curve for As I 2349.84 $\AA$. The net integral of the As I line divided by the net integral due to the Fe II lines at $2395.408 \AA$ and $2395.627 \AA$ is plotted. Precision bars are 1 standard deviation of 10 replicate measurements. Each measurement was obtained by averaging the results of 100 spectra where each spectrum was taken from a different point on the sample. 


\section{As I 2349.84Å Calibration Curve}

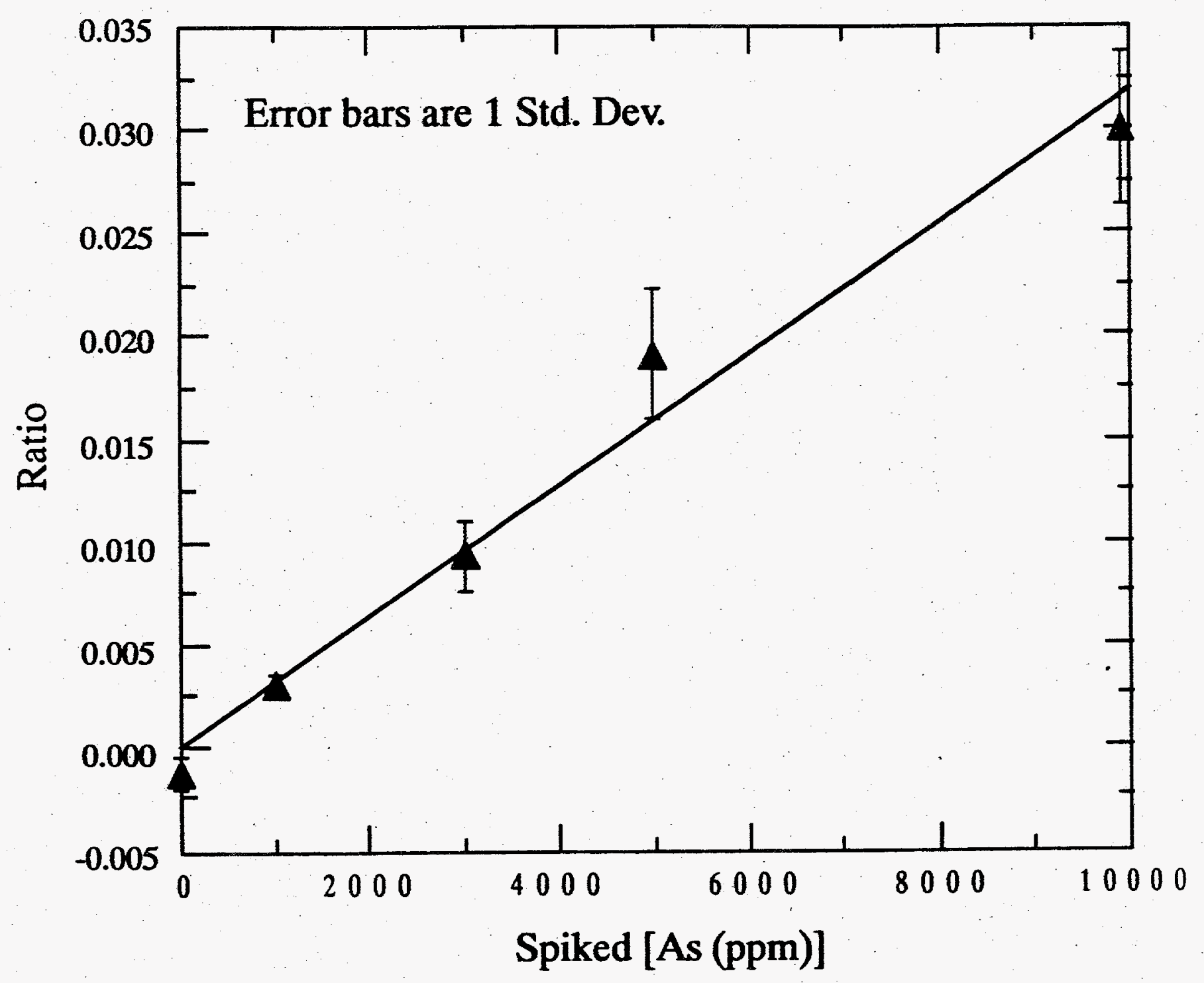




\section{CHROMIUM}

The most sensitive lines we have identified for LIBS detection of chromium in soil are Cr I 4254.35 $\AA$ and Cr I 3593.49 $\AA$. Detection limits for these two lines are $23 \mathrm{ppm}$ and $28 \mathrm{ppm}$, respectively, based on three standard deviations of the blank.

Figure 7 is the LIBS spectrum for a sample spiked with chromium to produce a concentration of $41.4 \mathrm{ppm}$ (+ background level). There are no resolvable spectral interferences for the $\mathrm{Cr} I 4254.35 \AA$ line. A very weak shoulder due to titanium appears to the red. The figure also shows the two unresolved Fe I lines at 4250.12 and $4250.79 \AA$ which were used as internal reference for the calibration curve.

Figure 8 is a pair of calibration curves for the $\operatorname{Cr} I 4254.35 \AA$ line. Data for the two curves were acquired using different laser pulse energies. This was done to investigate the reduction in sensitivity which results from decreased irradiance. This transition of chromium is a resonance line with an excited state at $23,500 \mathrm{~cm}^{-1}$. The curvature in both calibration curves indicates self-absorption at high concentrations. Figure 9 shows linear fits to the same data using only those samples with concentrations below $225 \mathrm{ppm}$. The $3 \mathrm{~s}$ detection limits derived from these linear fits are $23 \mathrm{ppm}$ and $67 \mathrm{ppm}$ for laser pulse energies of $125 \mathrm{~mJ}$ and $20 \mathrm{~mJ}$, respectively. A similar reduction in sensitivity with lower laser pulse energy occurs without using the Fe internal reference. Relative standard deviations for the data points above the detection limit range from $20 \%$ to $31 \%$ for a laser pulse energy of $20 \mathrm{~mJ}$ and from $17 \%$ to $37 \%$ for a laser pulse energy of $125 \mathrm{~mJ}$.

The calculated concentration of the blank using standard additions is $32 \mathrm{ppm}$ and $46 \mathrm{ppm}$ for the $125 \mathrm{~mJ}$ and $20 \mathrm{~mJ}$ data, respectively. The value obtained by laboratory $\mathrm{X}$-ray fluorescence spectroscopy was $13.7 \pm 9.0$ based on two measurements [5].

A similar set of data was taken for the $\mathrm{Cr}$ I lines in the $3600 \AA$ region. Figure 10 is a spectrum for chromium spiked soil in this spectral region. Three lines appear in this region: $\operatorname{Cr} I 3578.69 \AA, \operatorname{Cr} I 3593.49 \AA$, and $\operatorname{Cr} I$ 3605.33 $\AA$. The best results were obtained with the line at $3593.49 \AA$. Not uncoincidentally, this is the line with the least amount of spectral interference. $3 \mathrm{~s}$ detection limits using this line were $28 \mathrm{ppm}$ for data using $125 \mathrm{~mJ}$ of laser pulse energy and $93 \mathrm{ppm}$ for data taken with $20 \mathrm{~mJ}$ of laser pulse energy. Relative standard deviations for samples above the detection limit range from $8 \%$ to $21 \%$ for the $125 \mathrm{~mJ}$ data and $13 \%$ to $24 \%$ for the $20 \mathrm{~mJ}$ data. 
Figure 7. LIBS spectrum of soil near Cr I $4254.35 \AA$. The upper trace was obtained from a sample spiked with chromium. The net concentration of the spiked soil is 41.4 ppm plus background. The lower trace is the spectrum obtained from a soil blank. 


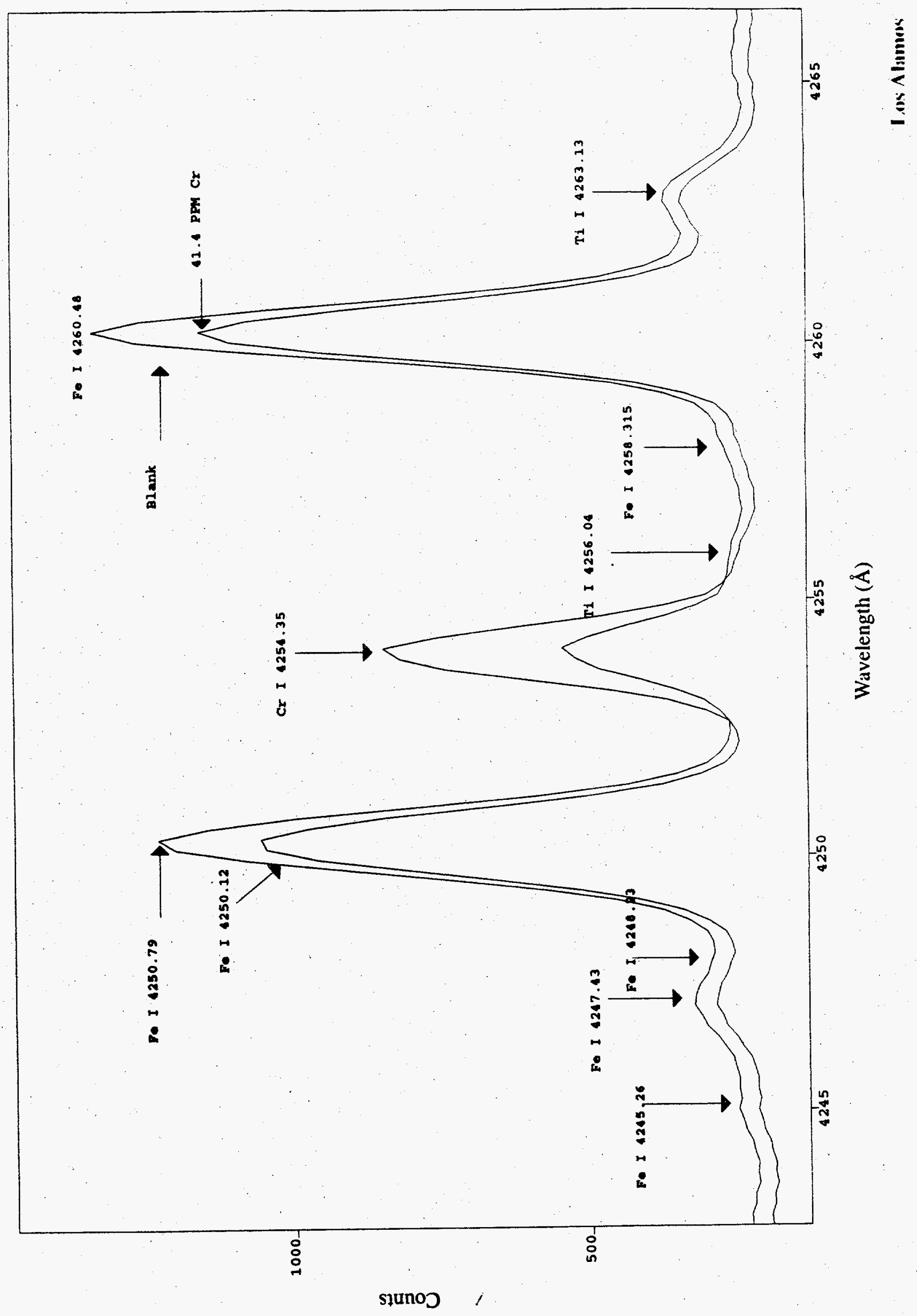


Figure 8. Calibration curves for $\mathrm{Cr} I 4254.35 \AA$. The net integral of the $\mathrm{Cr}$ I line divided by the net integral due to the two unresolved Fe I lines at $4250.12 \AA$ and $4250.79 \AA$ is plotted. Precision bars are 1 standard deviation of 10 replicate measurements. Each measurement was obtained by averaging the results of 100 spectra where each spectrum was taken from a different point on the sample. 


\section{Cr@4254.35Ă CALIBRATION CURVE vs. LASER PULSE ENERGY}

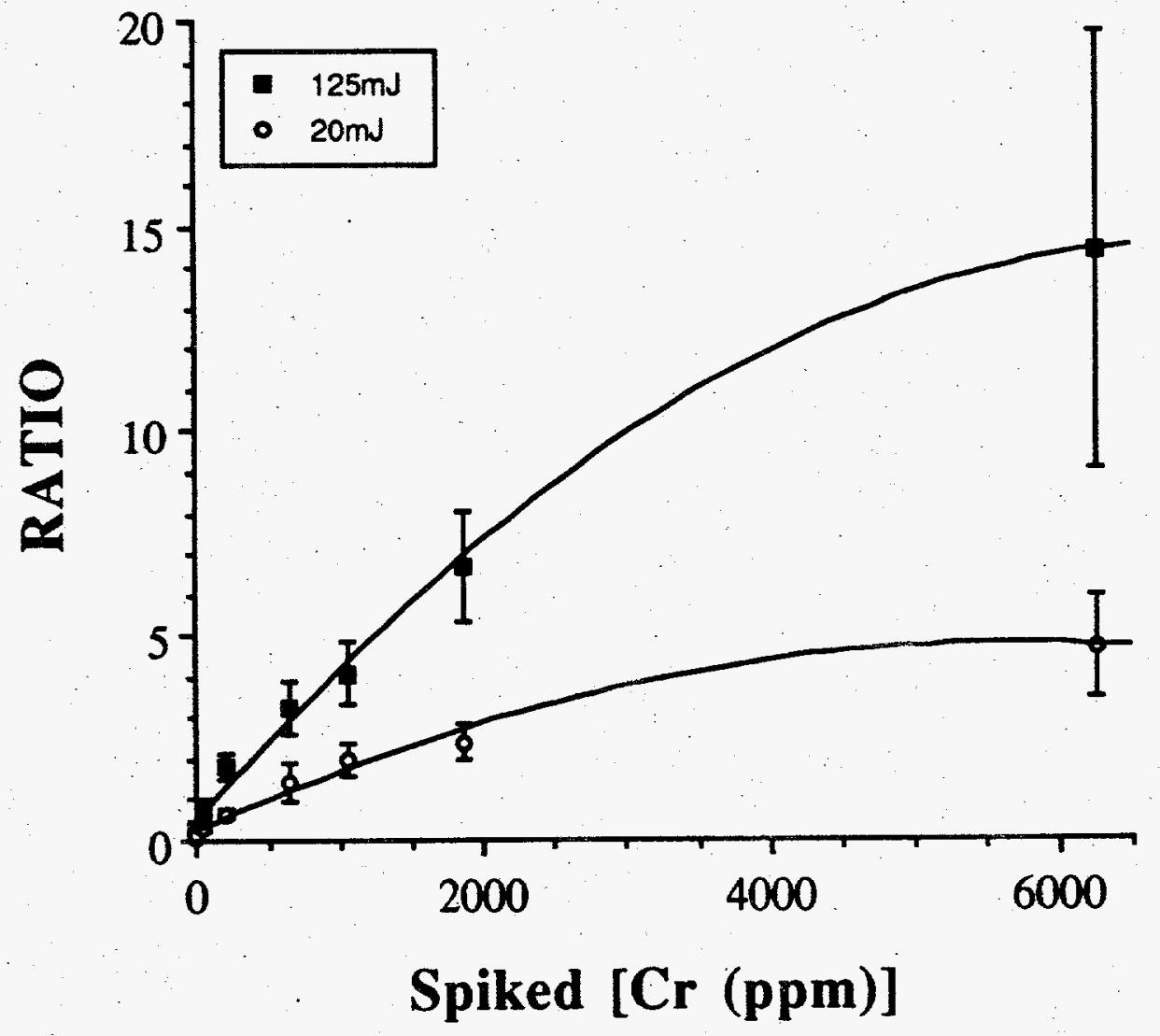


Figure 9. Calibration curves for Cr I 4254.35 $\AA$. This is an expanded view of the calibration curve presented in Figure 8. The lines are least squares fits to the data below 250 ppm Cr. 


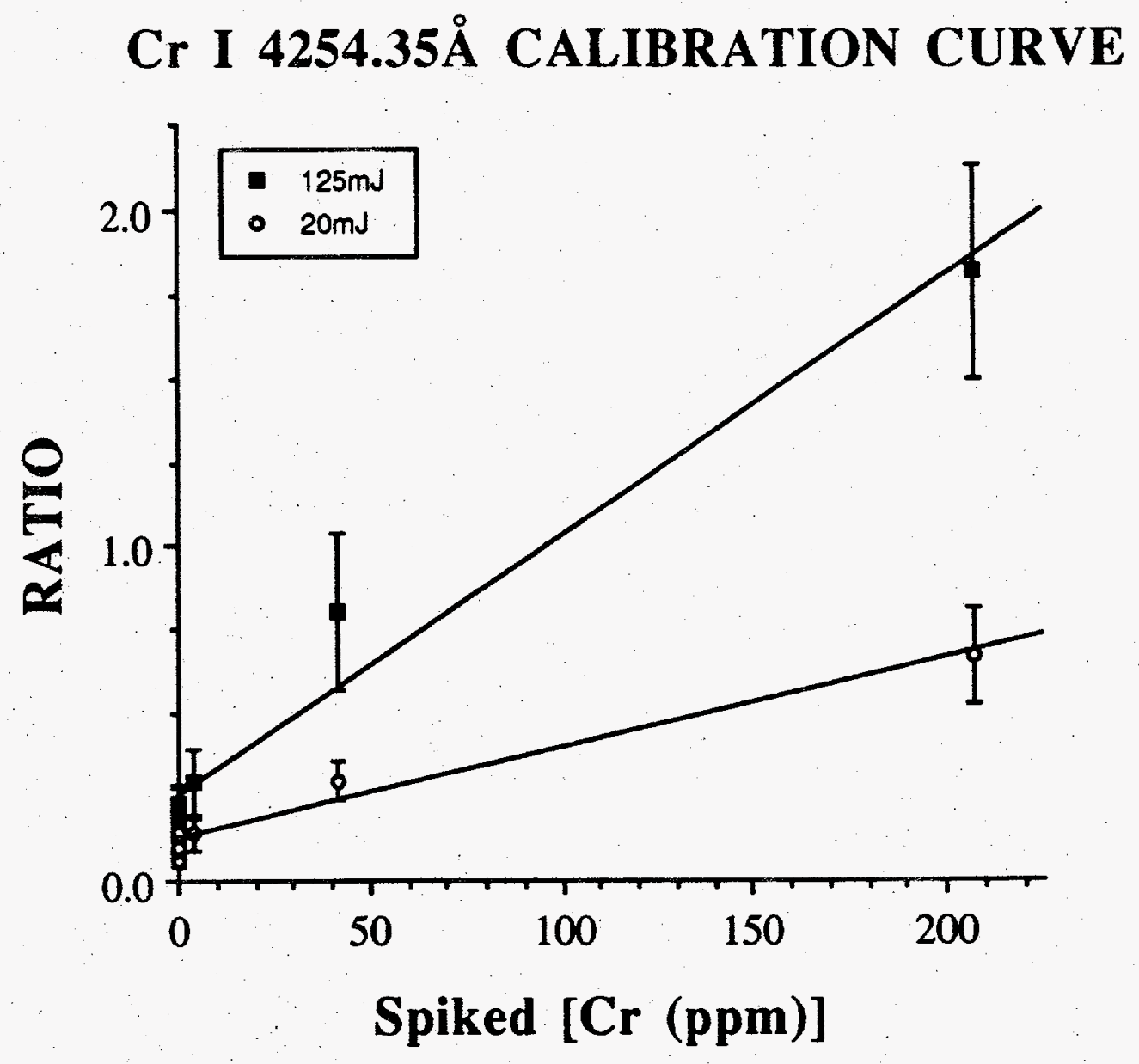


Figure 10. LIBS spectrum of soil near $3580 \AA$. The upper trace was obtained from the same sample as that used to obtain Figure 7. The lower trace is the spectrum obtained from a soil blank. 


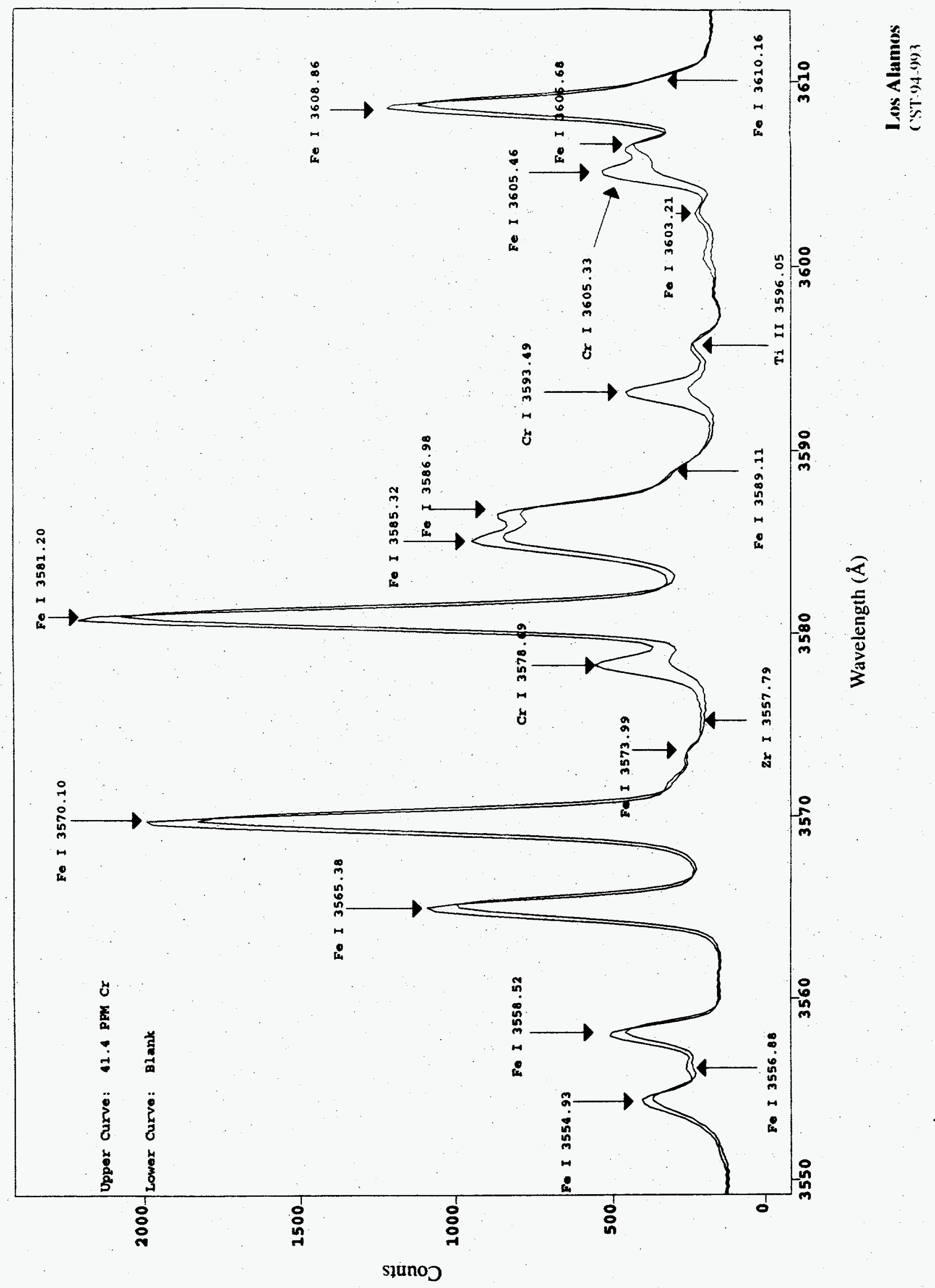




\section{CADMIUM}

The strongest line listed in the NIST tables is Cd I 6438.47 $\AA$. A LIBS spectrum of this region showed that $\mathrm{Ca} I 6439.07 \AA$ strongly interferes when spectral resolution is $2 \AA$. The Ca I line persists for $100 \mu$ s so using time discrimination will not aid in eliminating this interference. A $100 \mathrm{ppm}$ Cd soil sample could not be discerned on the side of the Ca peak. The other peaks which would appear in this region--6359.98 $\AA$, $6354.72 \AA$ and $6464.94 \AA$--are Cd II transitions and were not observed.

There are a number of other slightly weaker spectral lines, all of equal intensity in the NIST tables. We chose the $5085.822 \AA$ line to construct a calibration curve. This line is atomic and as a rule of thumb it is often easier to detect atomic transitions with LIBS. Also, this line is in the visible and detector sensitivity can be expected to be higher in this region. Figure 11 is a spectrum of $500 \mathrm{ppm}$ Cd in soil. There is some spectral interference from titanium at $5085 \AA$.

Figure 12 is a calibration curve for Cd I $5085.822 \AA$ using Fe I $5110.357 \AA$ as an internal reference. The bounds used to calculate the net integral for cadmium encompass both the Ti I 5085.33 $\AA$ and Ti I 5087.07 $\AA$ lines. Fluctuations in these titanium lines could result in elevated detection limits. The $3 \mathrm{~s}$ detection limit using this calibration curve is $73 \mathrm{ppm}$. This value is limited by the noise in the emission from the plasma. Relative standard deviations for each sample range from $10 \%$ to $19 \%$ for concentrations greater than the detection limit.

The titanium interference underlying the $\mathrm{Cd}$ line results in an apparent high level of $\mathrm{Cd}$ in the blank. We measure an apparent level of $176 \mathrm{ppm}$ compared to the general value of $\leq 2 \mathrm{ppm}$ for Los Alamos soil determined by X-ray fluorescence spectroscopy. A multivariate calibration could help reduce these effects of titanium interference.

A common technique used in this laboratory to decrease the effect of sample inhomogeneity is to use a "long spark". To produce the long spark, a cylindrical lens is used instead of a spherical lens to focus the laser beam onto the soil surface. As long as the irradiance is the same in both cases, the longer spark helps reduce the effect of sample inhomogeneities in the size range of $100 \mu \mathrm{m}$ to $1 \mathrm{~mm}$. Because of limitations in the pulse energy achievable in the laser used for this work, the irradiance could not be kept as high as that for the spherical focusing lens. The resulting detection limit using a cylindrical lens, $141 \mathrm{ppm}$ reflects the reduced irradiance.

The commonly used ICP lines $2144.9 \AA, 2288.0 \AA$ and $2265.0 \AA$ are inappropriate for this application. The Cd II $2144.9 \AA$ line is interfered by high iron concentrations, like those found in soils [3]. The Cd I 2288.0A line should not be used with high arsenic concentrations. The limitation on this line may not be severe if it is known from other data that the arsenic concentration is low. Finally, the Cd II 2265.0 line has a weak interference from the aluminum doublet at $2269 \AA$.

The facilitators for this project have recently requested that we try to improve the detection limit for cadmium to about $10 \mathrm{ppm}$. We are investigating other cadmium emission lines for this purpose. 
Figure 11. LIBS spectrum of soil near Cd I 5085.82A. The upper trace was obtained from a sample spiked with cadmium. The net concentration of the spiked soil is $298.99 \mathrm{ppm}$ plus background. The lower trace is the spectrum obtained from a soil blank. 


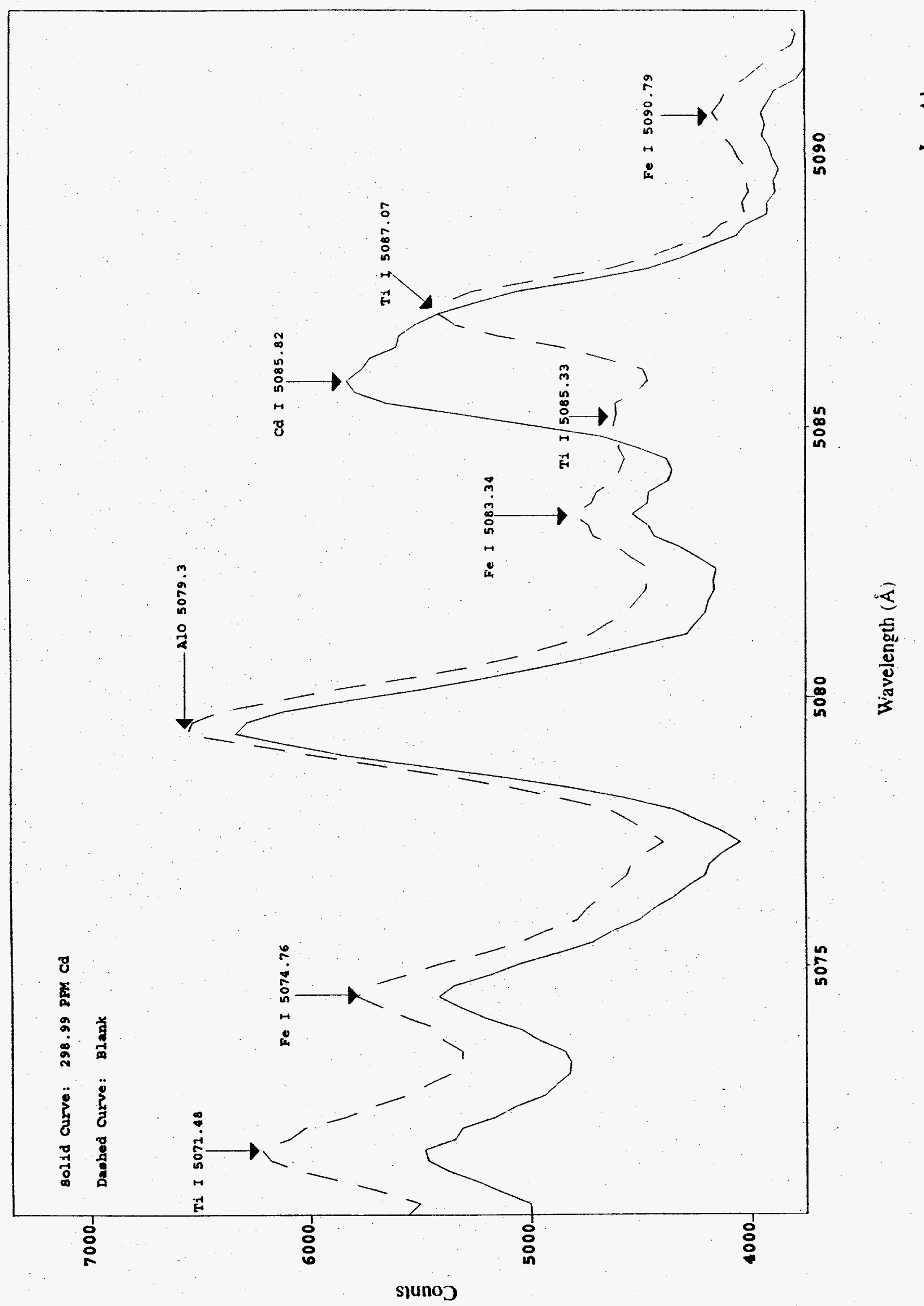


Figure 12. Calibration curve for Cd I 5085.82 $\AA$. RATIO is quotient of the net integral of the $\mathrm{Cd} I$ line divided by the net integral for Fe I.5110.357 $\mathrm{A}$. Precision bars are 1 standard deviation of 10 replicate measurements. Each measurement was obtained by averaging the results of 100 spectra where each spectrum was taken from a different point on the sample. 
Cd I 5085.82A CALIBRATION CURVE

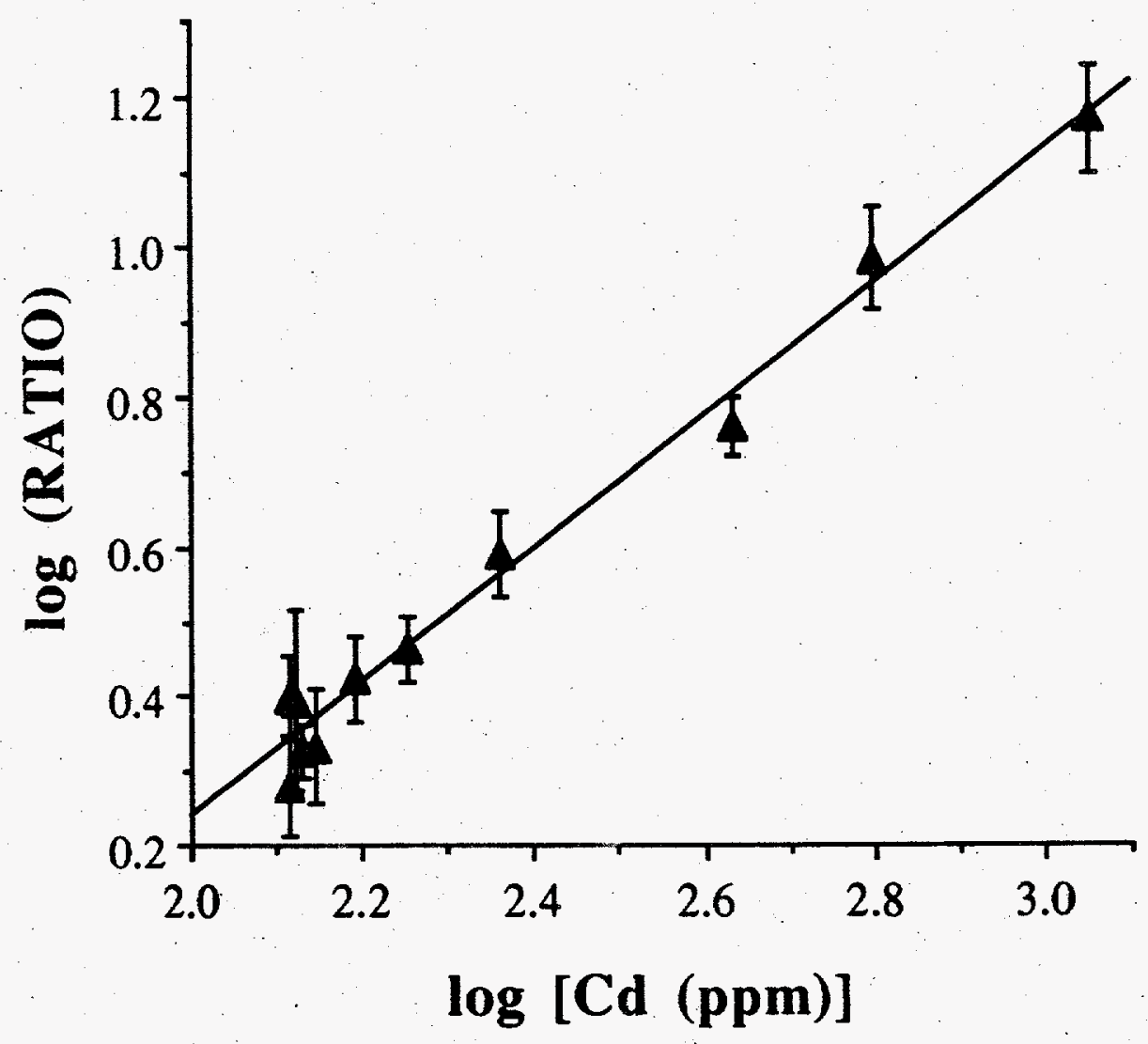


Figure 13 is a spectrum of a $100 \mathrm{ppm} \mathrm{Pb}$ sample (+ background concentration) in the neighborhood of the $4057.81 \AA$ line. The line has nearby interferences from manganese on both the blue side and, more severely, on the red side. The strength of the line however overcomes the interference and results in a good detection limit. Using the Fe I lines at $3997.392 \AA$ and at $3998.053 \AA$ as an internal reference, the 3 s detection limit was found to be $15 \mathrm{ppm}$. Using Fe I at $4063.6 \AA$ the $3 \mathrm{~s}$ detection limit was $17 \mathrm{ppm}$. Relative standard deviations for samples with concentrations from the detection limit to $1005 \mathrm{ppm}$ are in the range $7 \%$ to $19 \%$ for the data using the Fe I $4063 \AA$ reference line. RSDs for the data using the Fe I lines near $3998 \AA$ are in the range of $8 \%$ to $17 \%$. The difference in precision between the two data sets is probably insignificant. The calculated background concentration is about $75 \mathrm{ppm}$ using standard additions. The specific soil used in these experiments was not analyzed for lead by X-ray fluorescence spectroscopy. However, in a survey of soils around Los Alamos the lead concentration was found to be from $18 \mathrm{ppm}$ to $56 \mathrm{ppm} \mathrm{[4].} \mathrm{The} \mathrm{higher} \mathrm{value} \mathrm{obtained} \mathrm{from} \mathrm{the} \mathrm{LIBS}$ data is probably due to the weak manganese interference found near the lead line. 
Figure 13. LIBS spectrum of soil near Pb I 4057.81 $\AA$. The trace with the largest lead peak was obtained from a sample spiked with lead. The net concentration of the spiked soil is $298.99 \mathrm{ppm}$ plus background. The trace with the lower concentration of lead is the spectrum obtained from a soil blank. 


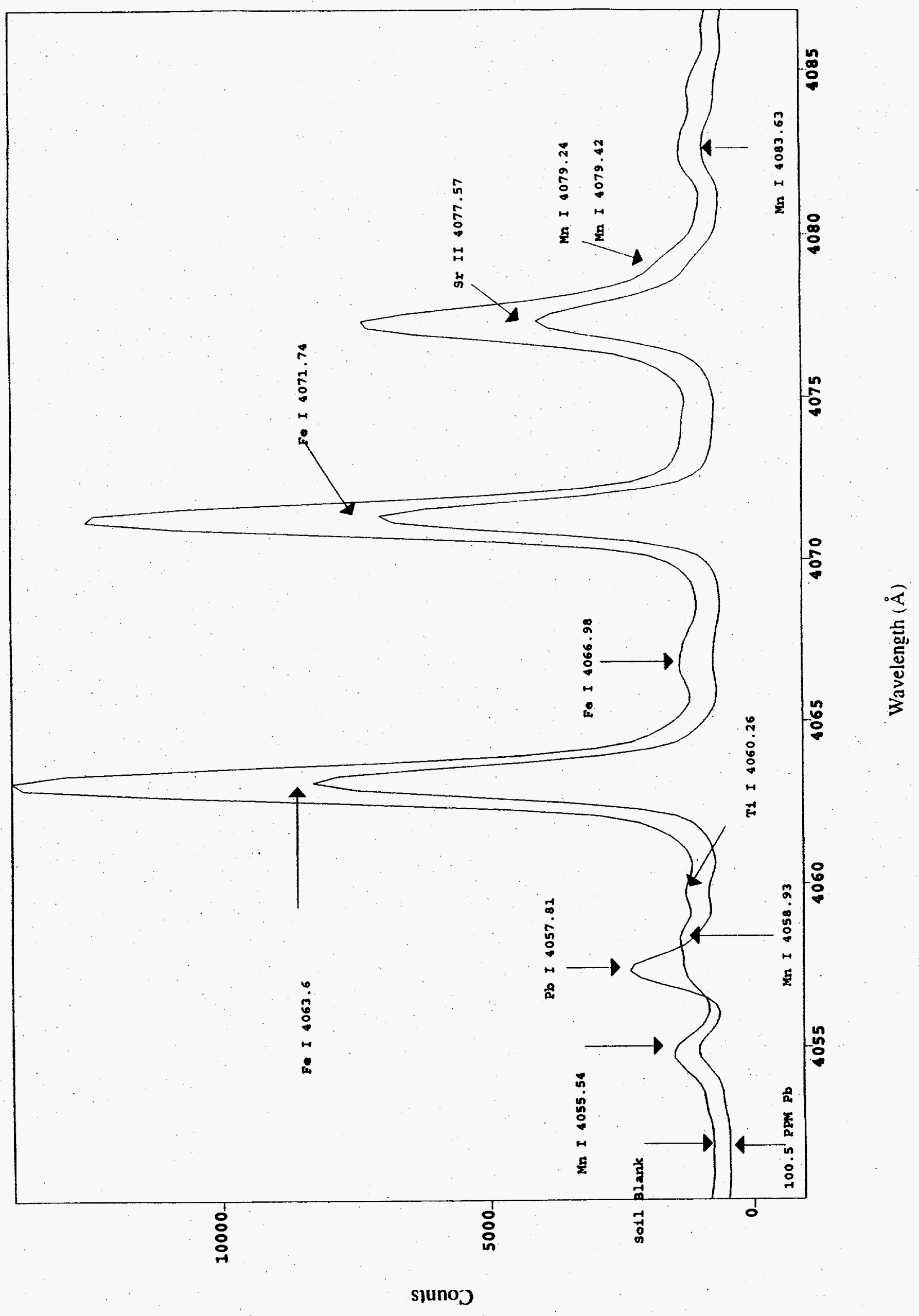




\section{STRONTIUM}

There are three strong strontium lines which show strong emission using LIBS. These are Sr II 4077.71 $\AA$, Sr II 4215.52 $\AA$ and SrI $4607.33 \AA$. Figures 14 through 16 show the LIBS spectra obtained from spiked soil at each of these wavelengths. The residual intensity in the blank is due to the background strontium level in the soil used. There is a possible very weak interference to the red of the $4077 \AA$ line. The $4215.52 \AA$ has a slight spectral interference from Fe I at $4217.56 \AA$. The $4607.33 \AA$ line also has a very weak, but unidentified, peak which overlaps on the red edge. In no case is the spectral interference particularly limiting. The calibration curves for these lines show curvature at high concentrations, indicative of the onset of self-absorption in the plasma. Below about $325 \mathrm{ppm}$ the calibration curves are effectively linear. Using these data the 3 s detection limits for $4077.71 \AA, 425.52 \AA$ and $4607.33 \AA$ are 30,24 and $80 \mathrm{ppm}$, respectively. The calculated level of the blank is 35,29 and $46 \mathrm{ppm}$ when using these three lines. The value obtained by XRF was $70.5 \pm 8.7 \mathrm{ppm}$ [5]. 
Figure 14. LIBS spectrum of soil near Sr II 4077.71 $\AA$. The upper trace was obtained from a sample spiked with strontium. The net concentration of the spiked soil is $25.4 \mathrm{ppm}$ plus background. The lower trace is the spectrum obtained from a soil blank. 


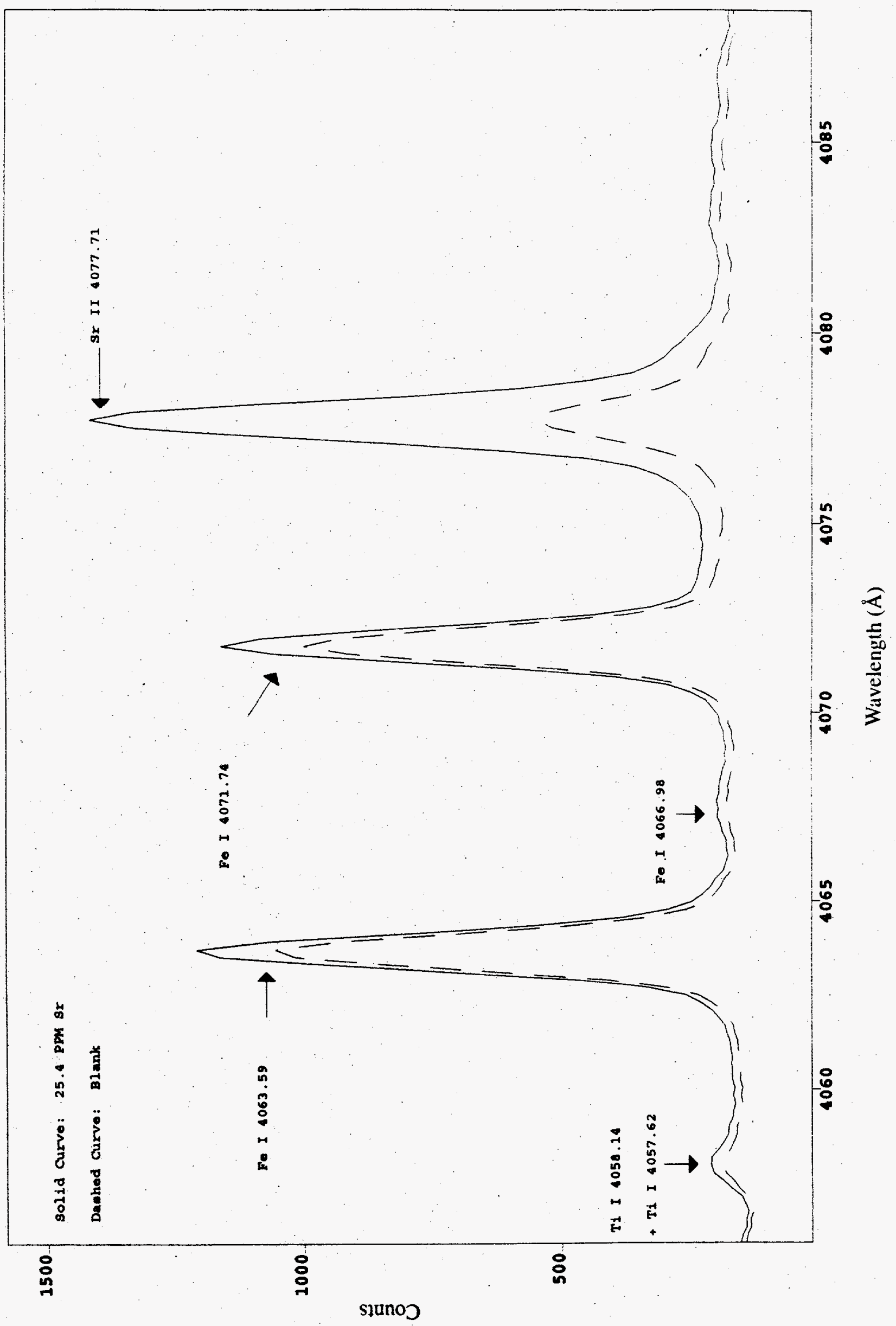


Figure 15. LIBS spectrum of soil near Sr II 4215.52A. The upper trace was obtained from a sample spiked with strontium. The net concentration of the spiked soil is 25.4 ppm plus background. The lower trace is the spectrum obtained from a soil blank. 


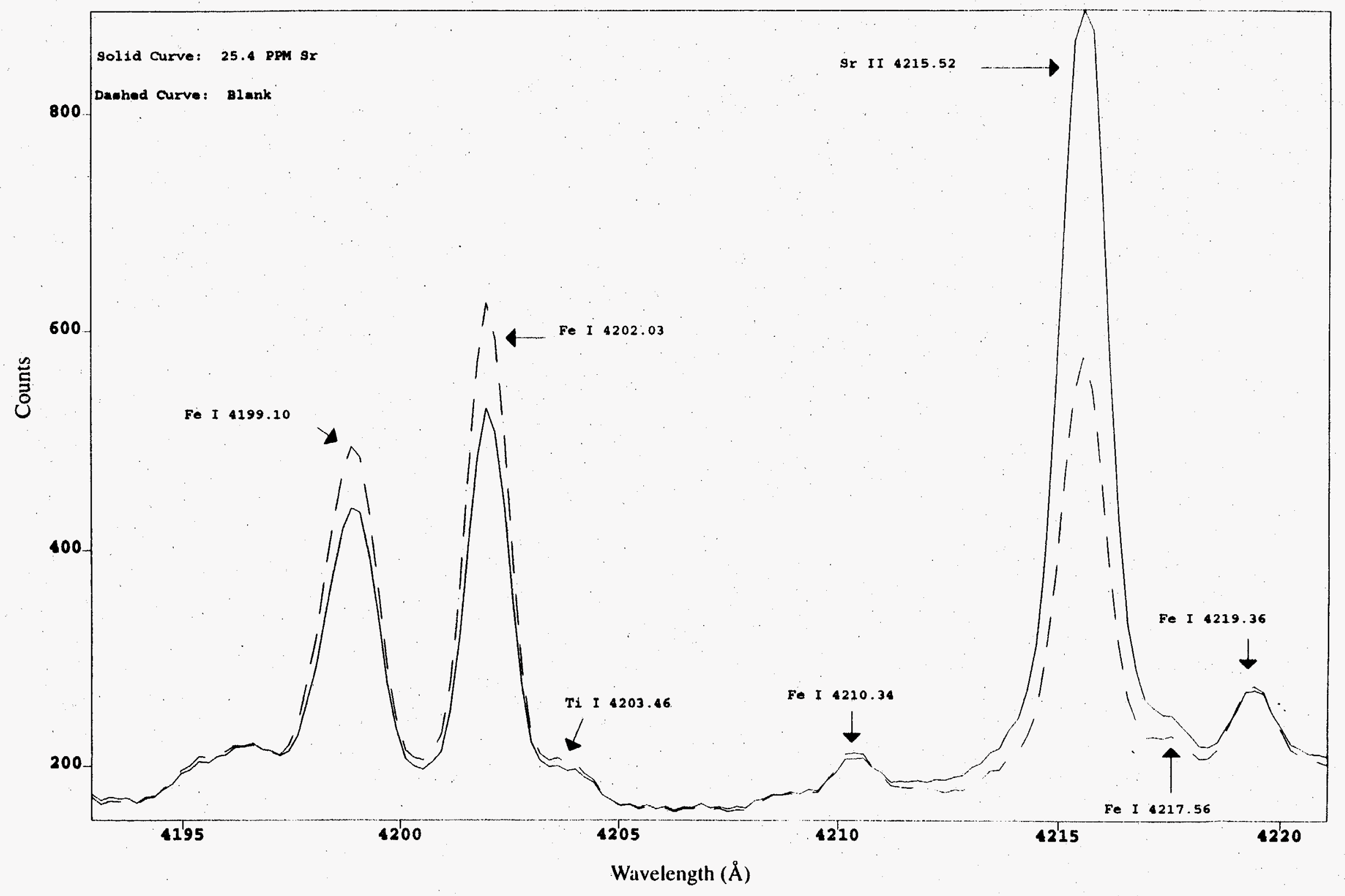

Los Alamos 


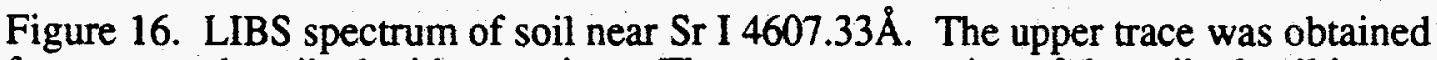
from a sample spiked with strontium. The net concentration of the spiked soil is $25.4 \mathrm{ppm}$ plus background. The lower trace is the spectrum obtained from a soil blank. 


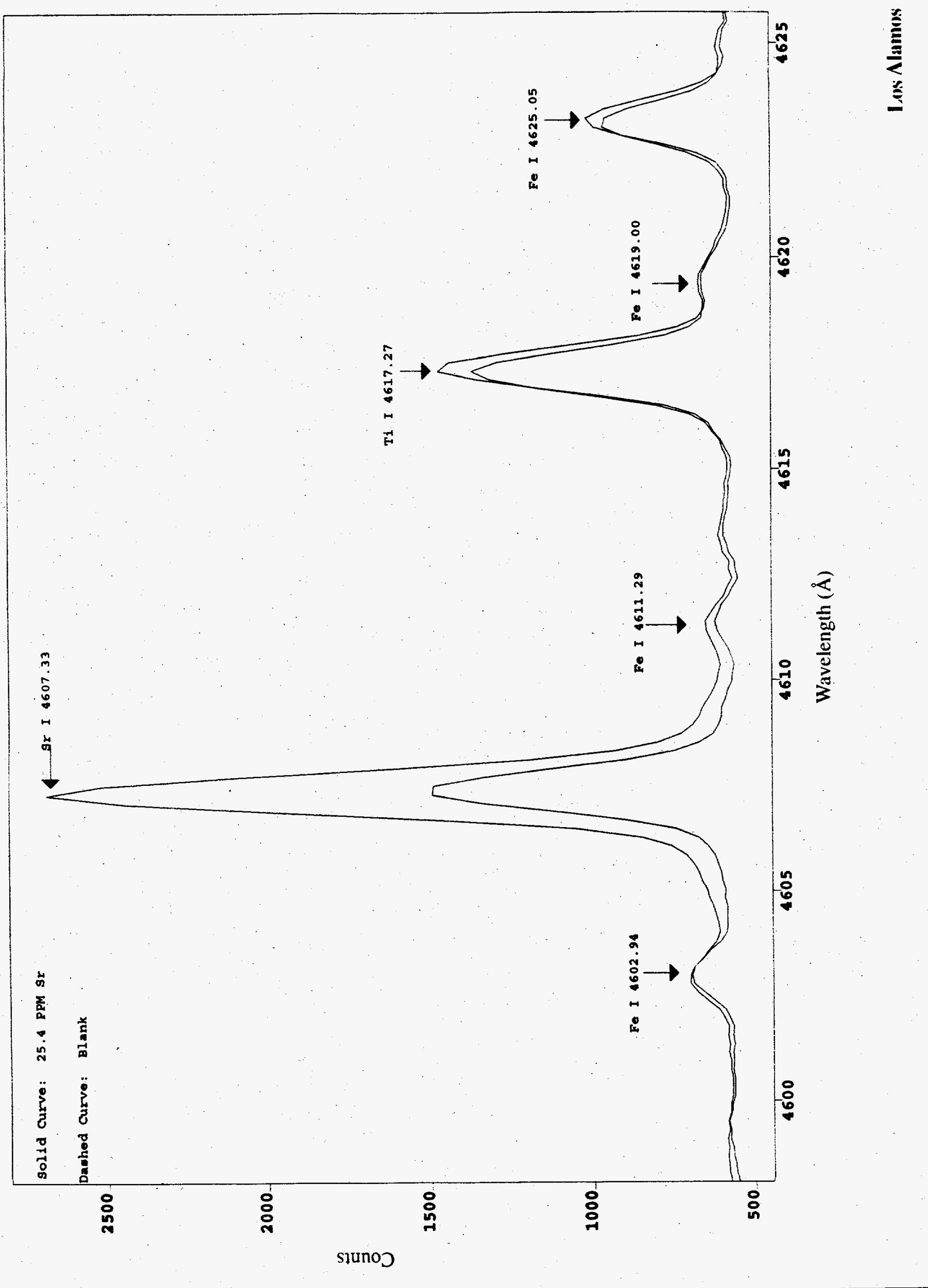




\section{BARIUM}

The data for barium was taken with soil samples of known composition. Soil samples were collected from many different areas and had very different compositions. Samples were pressed into pellets using $24 \mathrm{~g}$ of soil to $3 \mathrm{~g}$ of binder. The binder was a mix commonly used in X-ray fluorescence and has an elemental composition of $48.7 \%$ $\mathrm{C}, 42.6 \% \mathrm{O}, 8.1 \% \mathrm{H}$ and $0.6 \% \mathrm{~B}$. Figure 17 is a calibration curve for Ba II $4554.03 \AA$ using these pellets. The detection limit using this data is estimated to be $63 \mathrm{ppm}$ with a typical relative standard deviations of $5 \%$ for each data point. 
Figure 17. Calibration curve for Ba II $4554.03 \AA$. For this curve, no internal reference was used. 
Ba II 4554.03Å CALIBRATION CURVE

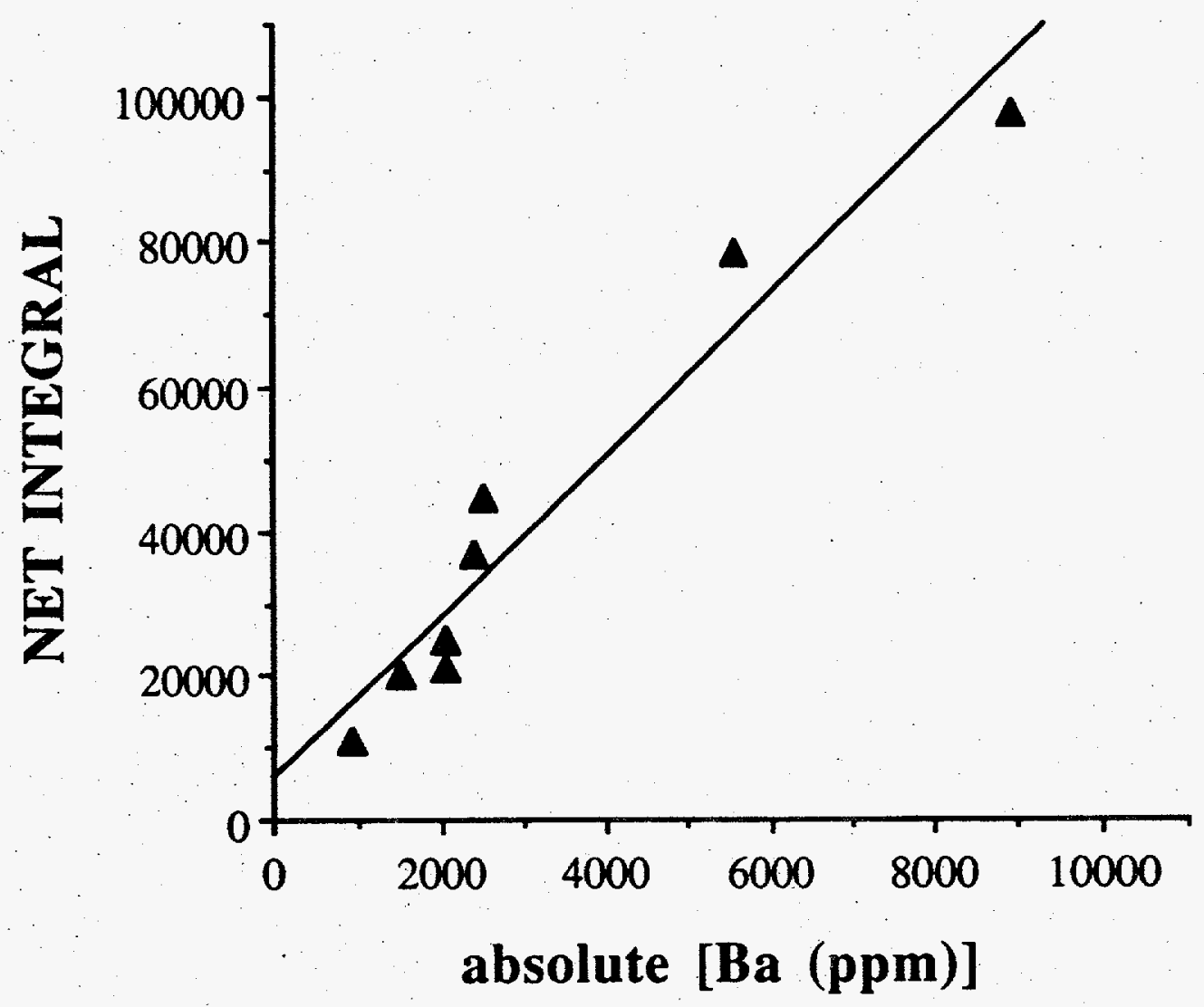




\section{BERYLLIUM}

The instrumentation used for measurements of beryllium in soils was different from that used for the other metals analyzed in this report. The laser used had a neargaussian spatial profile rather than a donut mode. The laser pulse energy used was the same as above and the focusing lens also had the same focal length. The detector was another intensified diode array. Time gating was used as for the other data. The spectrograph used had a resolution $1 / 2$ that of the one used in the rest of this work.

A calibration curve for beryllium is shown in Figure 18. Points on the calibration curve were calculated from six replicates of 100 laser shots each. The figure shows data for the unresolved $\mathrm{Be}$ II $3130.42 \AA$ and $3131.07 \AA$ lines. The Ca II $3179.33 \AA$ line was used as an internal reference. The calibration curve is linear over two orders of magnitude. Some curvature occurs above about $200 \mathrm{ppm}$. Using a linear fit to the points from samples with concentrations below $200 \mathrm{ppm}$, the $3 \mathrm{~s}$ detection limit is calculated to be $2 \mathrm{ppm}$. Precision ranges from 5\% to $36 \%$ with the lowest precision occurring near the detection limit. 
Figure 18. Calibration curve for Be II $3131 \AA$. RATIO is the quotient of the net integral of the Be II line divided by the net integral for Ca II 3179.33A. Precision bars are 1 standard deviation of 6 replicate measurements. Each measurement was obtained by averaging the results of 100 spectra where each spectrum was taken from a different point on the sample. 
Be II 3131Å CALIBRATION CURVE

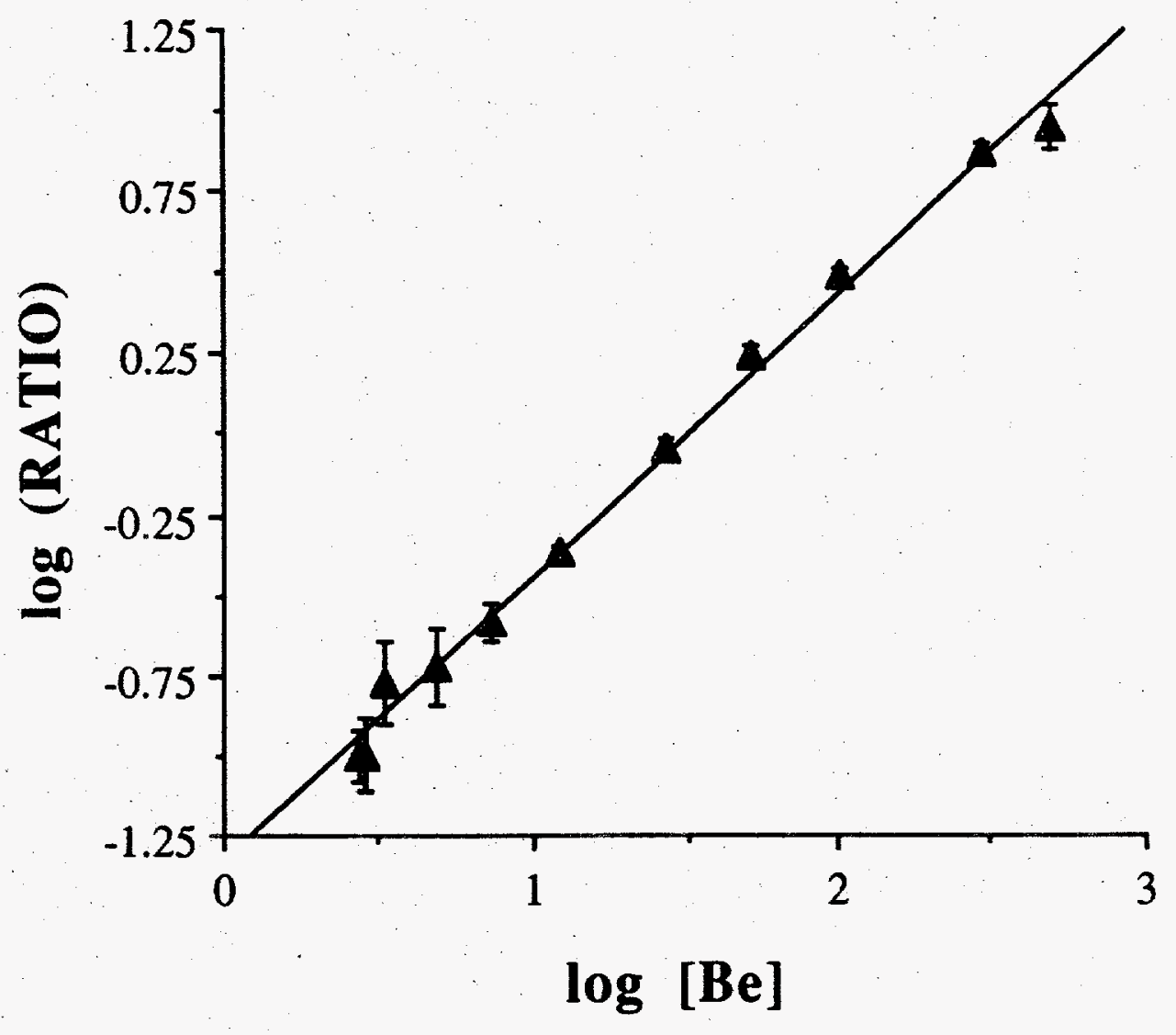




\section{CESIUM}

Some preliminary work has been done on cesium. The moderately strong line of Cs II at $4526.74 \AA$ was sought and could not be found. The strongest line of the atomic species at $6973.297 \AA$ was observed along with weaker lines at $6870.46 \AA$ and $6983.49 \AA$. The Cs I signal is clearly evident at a spiked Cs concentration of $500 \mathrm{ppm}$. Using standard additions, the cesium concentration in the blank is estimated to be $35 \mathrm{ppm}$. Measurements to develop a full calibration curve at this wavelength and a search for the more intense Cs II lines will be conducted in the near future. 


\section{MERCURY}

The strongest mercury line listed in the NIST tables is Hg I 2536.52 $\AA$. Figure 19 shows the spectrum of a $1000 \mathrm{ppm} \mathrm{Hg}$ in a soil sample in the region of this line. Strong spectral interference is observed from the Fe II $2536.67 \AA$ and $2536.80 \AA$ lines. No signal due to mercury is observable.

The next strongest line for mercury is $\mathrm{Hg}$ I $4358.33 \AA$. Several interfering peaks occur at this wavelength in soil. The intensities of these interferences are weak, however. A calibration curve for mercury will be obtained for this wavelength in the near future. 
Figure 19. LIBS spectrum of soil near $\mathrm{Hg}$ I $2536.52 \AA$. The solid curve was obtained from a sample spiked with mercury. The net concentration of the spiked soil is

$999.3 \mathrm{ppm}$ plus background. The dashed curve is the spectrum obtained from a soil blank. 


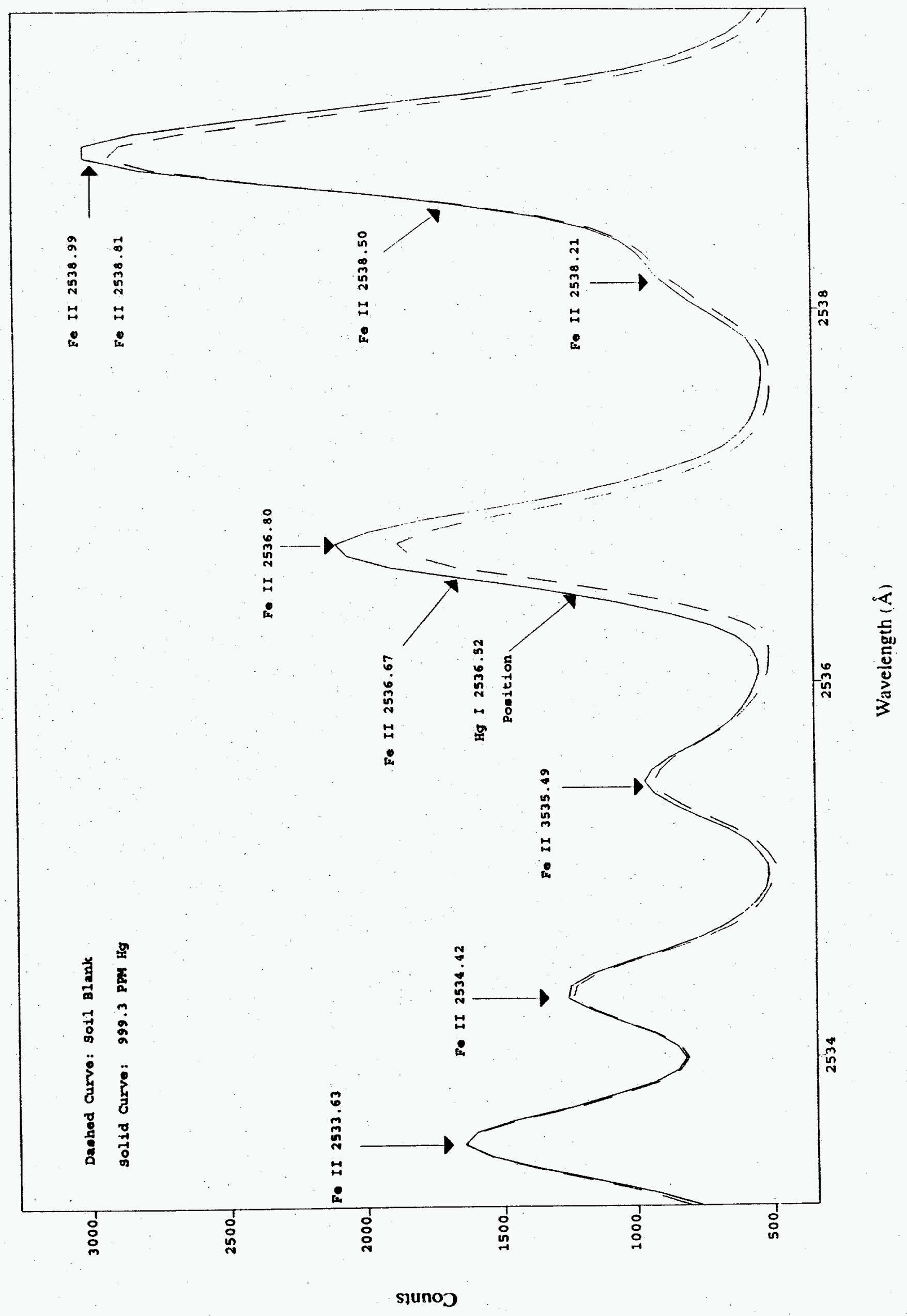




\section{SELENIUM}

The strongest selenium line suitable for a portable detector listed in the NIST table occurs at $2413.5 \AA$ and is an atomic line. As shown in Figure 20 , this line occurs on the side of an Fe II transition at 2413.31 . LIBS spectra taken at this wavelength for samples of with different selenium concentrations up to $1002 \mathrm{ppm}$ show no signal attributable to selenium. In ICP-AES, two lines near $1960 \AA$ are used [3]. This wavelength is not detectable with the apparatus available to us. Another commonly used line is at $2039.9 \AA$, but this has a reported interference from Sb I 2039.77 $\AA$. The $2039.9 \AA$ line could be useful if correction for antimony can be made from other spectral information. This will be investigated in the near future. 
Figure 20. LIBS spectrum of soil near Se I 2413.5 $\AA$. The upper trace was obtained from a sample spiked with selenium. The net concentration of the spiked soil is $1002 \mathrm{ppm}$ plus background. The lower trace is the spectrum obtained from a soil blank. 


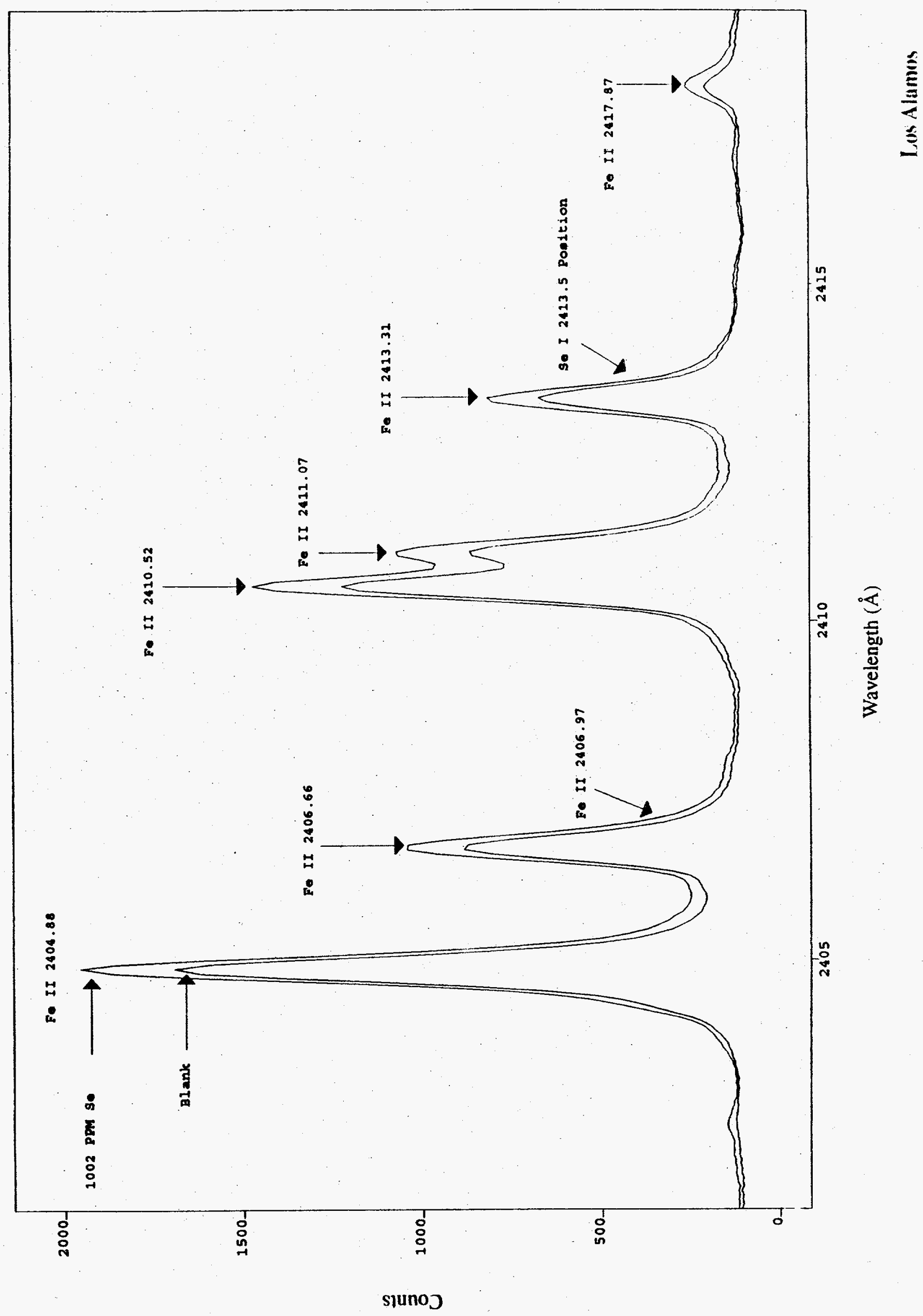




\section{ZIRCONIUM}

Zirconium, while not a RCRA metal, had been listed as an element of interest in the 1993 call for Technical Task Plan proposals.

Figure 21 is a spectrum of a 1000 ppm $\mathrm{Z}_{\mathrm{r}}$ in soil sample in the $3500 \AA$ region. Most of the zirconium lines show some spectral interference from iron. Calibration curves were constructed for several of the zirconium lines found between $3300 \AA$ and $3600 \AA$. The best lines of those selected for further study were the $\mathrm{Zr}$ II $3438.23 \AA$ and the $\mathrm{Zr}$ II $3572.47 \AA$ line. Detection limits were $103 \mathrm{ppm}$ and $86 \mathrm{ppm}$, respectively, with relative standard deviations ranging from $13 \%$ to $20 \%$ and $15 \%$ to $19 \%$ for the $3438 \AA$ and the $3572 \AA$ lines, respectively. The large number of lines available in a single spectrum and the varying degrees of spectral overlap with iron lines suggest a multivariate calibration will greatly improve the results for this analyte. 
Figure 21. LIBS spectrum of zirconium spiked soil near $3400 \AA$. The net concentration of the spiked soil is $100 \mathrm{ppm}$ plus background. 
Counts

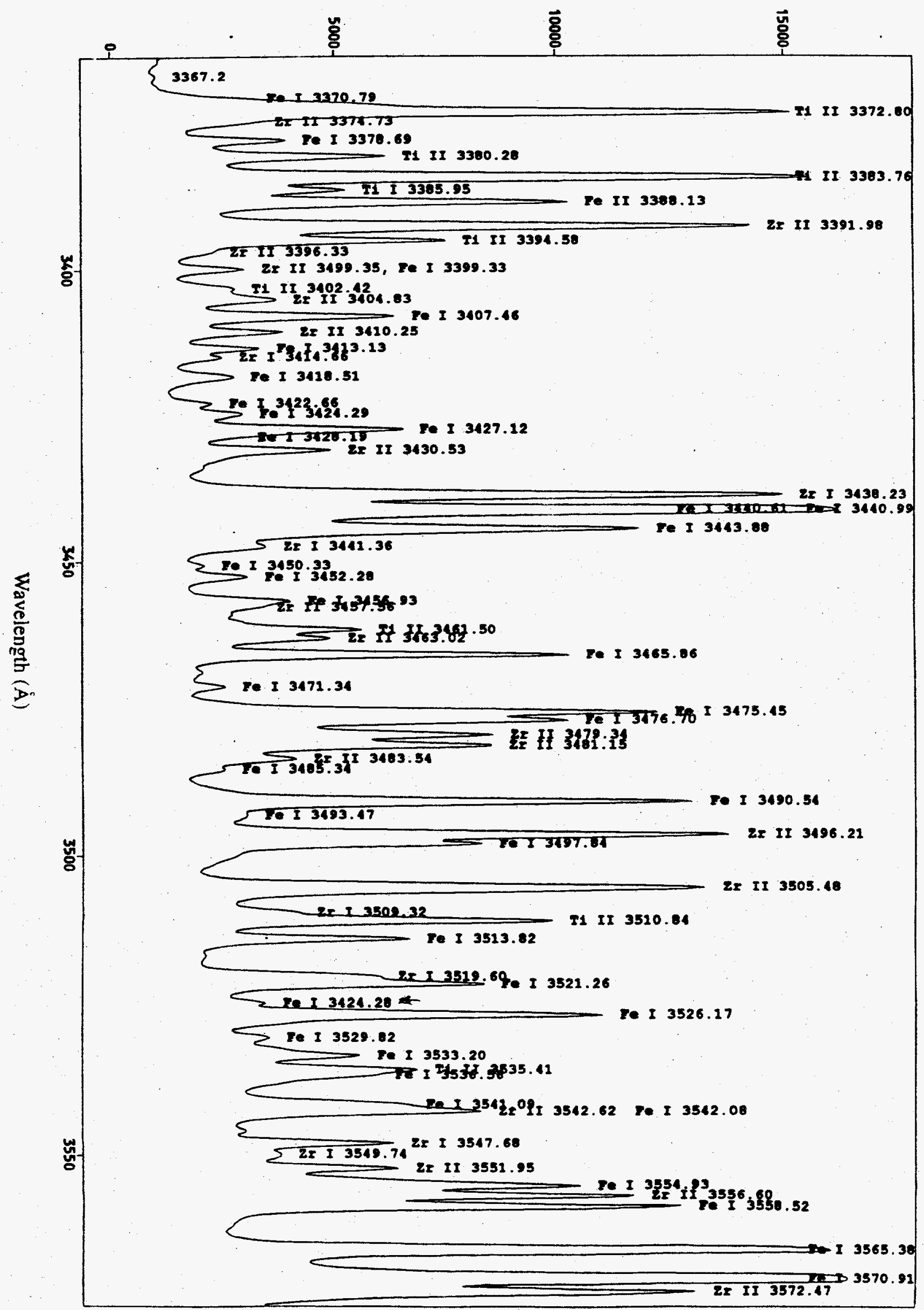




\section{ANTIMONY}

Many antimony lines have been investigated. The three lines studied in detail to

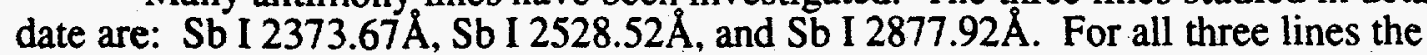
calibration curves have a weakly negative slope as a function of increasing concentration. Negatively sloped calibration curves can occur, in principle, for analyte lines with high oscillator strength, due to self-absorption. It is unlikely that this explanation is valid for these antimony lines since the oscillator strengths are known to be low. The effect is the same whether the solution matrix from which the samples are prepared contains $\mathrm{HCl}$ or $\mathrm{HNO}_{3}$. It is also the same at $2528.52 \AA$ when a curve is prepared using the data obtained for the three NIST standard reference soils.

We plan to acquire data for Sb I $2311.47 \AA$ to determine whether the effect is due to chemistry in the plasma. All three of the lines described above have some spectral interference from elements present in the soil matrix. If antimony is coupled through some reaction path to these elements, then the intensity contribution from the interfering elements may decrease with increasing antimony concentration. The line at $2311.47 \AA$ appears to be relatively free of spectral interference and may give better results. 


\section{REFERENCES}

1. D.A. Cremers and L.J. Radziemski, "Laser Plasma for Chemical Analysis," in Laser Spectroscopy and Its Applications (Marcel Dekker, New York, 1986) edited by L.J. Radziemski, R.W. Solarz, and J.A. Paisner. Chapter 5.

2. Wavelengths and Transition Probabilities for Atoms and Atomic Ions. Pan L. Wavelengths Joseph Reader and Charles H. Corliss, National Bureau of Standards.

3. CRC Handbook of Inductively Coupled Plasma Atomic Emission Spectroscopy, Asha Varma, CRC Press, 1991.

4. Clarence Duffy and Patrick Longmire, "Background Elemental Concentrations in Bandelier Tuff and Selected Soil Series, 1993," draft report, LANL, 1993.

5. Data provided by the Earth and Environmental Sciences Division, EES-1, Los Alamos National Laboratory, 1994. 